\title{
Stability of quantized time-delay nonlinear systems: a Lyapunov-Krasowskii-functional approach
}

\author{
Claudio De Persis · Frédéric Mazenc
}

Received: 26 September 2008 / Accepted: 24 May 2010 / Published online: 8 June 2010

(C) The Author(s) 2010. This article is published with open access at Springerlink.com

\begin{abstract}
Lyapunov-Krasowskii functionals are used to design quantized control laws for nonlinear continuous-time systems in the presence of constant delays in the input. The quantized control law is implemented via hysteresis to avoid chattering. Under appropriate conditions, our analysis applies to stabilizable nonlinear systems for any value of the quantization density. The resulting quantized feedback is parametrized with respect to the quantization density. Moreover, the maximal allowable delay tolerated by the system is characterized as a function of the quantization density.
\end{abstract}

Keywords Nonlinear systems · Time-delay systems · Quantized systems · Switched systems · Hysteresis

\section{Introduction}

Quantized control systems [3,12] are systems in which the control law is a piece-wise constant function of time taking values in a finite set. The design of quantized control systems is based on a partition of the state space. One value of the control law is associated to each set of the partition, and whenever the state crosses the boundary

\footnotetext{
C. De Persis $(\varangle)$

Faculty of Engineering Technology, Universiteit Twente, Enschede, The Netherlands

e-mail: C.DePersis@ctw.utwente.nl

C. De Persis

Dipartimento di Informatica e Sistemistica, Sapienza Università di Roma, Via Ariosto 25, 00185 Rome, Italy

F. Mazenc

Projet INRIA DISCO, CNRS-Supélec, 3 rue Joliot Curie, 91192 Gif-sur-Yvette, France

e-mail: Frederic.Mazenc@1ss.supelec.fr
} 
between two sets of the partition, the control law takes the new value associated to the set which the state has just entered.

When dealing with the problem of stabilizing the origin of the state space for linear discrete-time systems, the paper [3] has shown the effectiveness of logarithmic quantization in which the partition of the state space is coarser away from the origin and denser in its vicinity. It has also introduced the notion of quantization density, that is the number of regions of the partition per unit of space. Intuitively, the larger is the quantization density, the easier is the quantized control problem, since as the quantization density gets larger, the quantized control law approaches a control law without quantization. The paper [12] deals with a similar problem but for nonlinear continuous-time systems which can be made input-to-state stable with respect to the quantization error. Recently, the paper [1] has investigated quantized control systems in the framework of discontinuous control systems, discussing appropriate notions of solutions, namely Krasowskii and Carathéodory solutions. In this framework, the effect of quantization is viewed as an additional disturbance whose effect is attenuated by a Lyapunov redesign of the control law. Namely, given any nonlinear continuoustime process which is stabilizable by a continuous feedback, and given any value of the quantization density, it is always possible to find a new feedback depending on the quantization density, in such a way that the process in closed-loop with the quantized control law is practically stable with a basin of attraction which can be made arbitrarily large. Other notions of robustness (namely, robustness in the sense of the $\mathcal{L}_{2}$-gain) in connection with quantized control problems have been examined in [5] and [1]. Moreover, in the former, an adaptive quantized control scheme has been investigated.

Since quantized controls take values in a finite set, they lend themselves to be implemented over a finite data-rate communication channel. Data transmitted over a channel are usually delivered at the other end of channel after a delay. The problem of quantized control systems in the presence of delays then arises very naturally. Such a problem has been examined for the first time in [13], where the connection between Razumikhin-type theorems and the ISS small-gain theorem established in [20] was exploited. In recent years, besides [20], other contributions in the area of nonlinear time-delay systems have appeared (see, for instance, [4,8,9,14-18] and references therein). In particular, the paper [14] has proposed a Lyapunov-Krasowskii-functional approach to study the stabilizability of nonlinear systems in the presence of a delay in the input.

The aim of this paper is to pursue the approach of [14] in the analysis and design of quantized time-delay control systems. Besides the use of Lyapunov-Krasowskii functionals, there are other important features of the approach which make our paper different from other contributions. We implement the quantized control with the hysteretic mechanism suggested in [5] to avoid chattering. It is known from [2] that, in the case no delay is present, the analysis of such hysteretic solutions can be reduced to the analysis of Krasowskii and Carathéodory solutions considered in [1]. In the case of quantized time-delay systems, the adoption of the hysteretic solution is desirable. First, because it allows us to avoid technical issues related to more general notions of solutions of time-delay quantized (that is, discontinuous) systems. Second, the existence of more general solutions such as Carathéodory solutions is guaranteed only under additional conditions (see, e.g. [1]). Another feature which is worth mentioning is that, as in [1], our analysis applies to stabilizable nonlinear systems for any value 
of the quantization density, provided that suitable conditions are satisfied. Then, the quantized feedback which stabilizes the closed-loop system despite the delay turns out to be parametrized with respect to the quantization density.

Our approach leads to a set of conditions to design quantized control systems which are robust with respect to delays. Since we employ the results of [14] based on Lyapunov-Krasowskii functionals, our conditions represent an alternative to the conditions derived using Razumikhin-like theorems in $[13,20]$. Other conditions could be derived using recent results on input-to-state stability of time-delay systems via Lyapunov-Krasowskii functionals $([4,18]$ where a few comments in this regard have been presented). However, this investigation is beyond the scope of the paper.

In the next section, we present a few preliminaries, such as the definition of the quantizer and the notion of solution we adopt. The main result along with the standing assumptions and a couple of examples are examined in Sect. 3. Proof of the main result is given in Sect. 4. Conclusions are drawn in Sect. 5.

\section{Notation, definitions}

- $\mathbb{R}_{\geq 0}$ (respectively, $\mathbb{R}_{>0}$ ) denotes the set of non-negative (positive) real numbers.

- Let $r_{1}, r_{2}$ be two real numbers such that $r_{1}<r_{2}$. Let $C^{1}\left(\left[r_{1}, r_{2}\right], \mathbb{R}^{m}\right)$ (respectively, $\left.\bar{C}^{1}\left(\left[r_{1}, r_{2}\right], \mathbb{R}^{m}\right)\right)$ denote the set of continuously differentiable (respectively, piecewise continuously differentiable) functions $\phi(\cdot):\left[r_{1}, r_{2}\right] \rightarrow \mathbb{R}^{m}$.

- Norms. $|\cdot|$ stands for the Euclidean norm, $\|\phi\|_{c}=\sup _{t \in\left[r_{1}, r_{2}\right]}|\phi(t)|$ stands for the norm of a function $\phi \in C^{1}\left(\left[r_{1}, r_{2}\right], \mathbb{R}^{m}\right)$.

$-\operatorname{sgn}(r), r \in \mathbb{R}$, denotes the sign function, i.e. the function $\operatorname{such}$ that $\operatorname{sgn}(r)=1$ if $r>0, \operatorname{sgn}(r)=-1$ if $r<0$, and $\operatorname{sgn}(r)=0$ if $r=0$.

- To simplify the notation, we will frequently use the notation of the Lie derivative. More precisely, if $f: \mathbb{R}^{n} \rightarrow \mathbb{R}^{n}$ is a vector field and $h: \mathbb{R}^{n} \rightarrow \mathbb{R}$ is a scalar function, we may use the notation $L_{f} h(x)$ for $\frac{\partial h}{\partial x}(x) f(x)$.

- A continuous function $k:[0, \infty) \rightarrow[0, \infty)$ is of class $\mathcal{K}$ provided it is zero at zero and strictly increasing. A class $\mathcal{K}_{\infty}$ function is a class $\mathcal{K}$ function which in addition is unbounded.

- We shall often omit arguments of functions to simplify notation.

- For a real-valued function $z(t)$, we denote by $z\left(t^{+}\right)$the right $\operatorname{limit}_{m>t, m \rightarrow t} z(m)$.

\section{Problem formulation}

We are interested in investigating the stability property of systems when the feedback control law undergoes quantization and delays. This problem arises in (idealized) scenarios in which a finite bandwidth channel lies in the feedback loop and introduces a delay. In the sub-sections below, we recall what is meant by quantization and what is a quantizer, we introduce the quantized time-delay system and the notion of solution we adopt, and finally the formulation of the problem.

\subsection{Quantizers}

To the purpose of describing our system in more formal terms, we introduce the following multi-valued map, which will be referred to henceforth as the quantizer. Let $u_{0}>0$ 
and $0<\rho<1$ be real numbers, let $u_{i}=\rho^{i} u_{0}$ and $U=\left\{0, \pm u_{i}, \pm u_{i}(1+\delta)^{-1}\right.$, $i=0,1, \ldots, j\}$, with $j \geq 1$ an integer. Let $\delta=(1-\rho)(1+\rho)^{-1}$ and

$$
\Psi(u)=\left\{\begin{array}{lrlrl}
u_{i} \operatorname{sgn}(u), & \frac{1}{1+\delta} u_{i} & <|u| \leq \frac{1}{1-\delta} u_{i}, & 0 \leq i \leq j \\
\frac{u_{i}}{1+\delta} \operatorname{sgn}(u), \frac{1}{(1+\delta)^{2}} u_{i} & <|u| \leq \frac{1}{(1+\delta)(1-\delta)} u_{i}, & 0 \leq i \leq j \\
0, & 0 \leq|u| \leq \frac{1}{1+\delta} u_{j} . &
\end{array}\right.
$$

A picture of the map is given in Fig. 1. Observe for later use that

$$
\rho=\frac{1-\delta}{1+\delta}
$$

and

$$
u_{i}=\left(\frac{1-\delta}{1+\delta}\right)^{i} u_{0}, \quad \forall i \in\{0,1, \ldots, j\}
$$

A few remarks are in order:

- The range of the quantizer, i.e. its interval of definition, is $\left[-\frac{u_{0}}{1-\delta}, \frac{u_{0}}{1-\delta}\right]$. We do not define $\Psi(u)$ for $|u|>\frac{u_{0}}{1-\delta}$, since we will design the parameter $u_{0}$ in such a way that the control $|u(t-\tau)|$, which is the actual argument of the map $\Psi$, never exceeds this upper bound.

- The logarithmic quantizer with a finite number of quantization levels, which is a truncated version of the quantizer with an infinite number of quantization levels, was introduced in [3, Sect. V], and it is as follows:

$$
\Psi(u)=\left\{\begin{array}{lrl}
u_{i} \operatorname{sgn}(u), & \frac{1}{1+\delta} u_{i}<|u| \leq \frac{1}{1-\delta} u_{i}, 0 \leq i \leq j \\
0, & 0 \leq|u| \leq \frac{1}{1+\delta} u_{j} .
\end{array}\right.
$$

Compared with (4), the quantizer (1) considered in this paper has additional quantization levels. To have a pictorial representation of the quantizer (4), one can refer to Fig. 1 and remove the quantization levels labeled as $\frac{u_{0}}{1+\delta}$ and $\frac{u_{1}}{1+\delta}$. The new quantization levels in (1) are added to avoid chattering. This will be explained in detail as soon as the system we are interested in and the notion of solution we adopt are introduced (see Remark 1 below).

- The parameter $\rho$ can be viewed as a measure of the quantization density, since the smaller is $\rho$, the coarser is the quantizer [3]. In fact, by (2), as $\rho$ approaches $0, \delta$ approaches 1, that is the width of the sector bound in Fig. 1 gets larger and, given an interval of fixed length on the $u$-axis in Fig. 1, $\Psi(u)$ will have fewer quantization levels as $u$ ranges over that interval.

- In the quantizer (1), the parameters $\delta, u_{0}, j$ appear. Throughout the paper, we shall assume that $\delta$ can take any value in the interval $(0,1)$ (i.e. the quantization density can be equal to any value). On the other hand the positive real number $u_{0}$ (which defines the range of the quantizer) and the integer $j$ (which gives the number of 


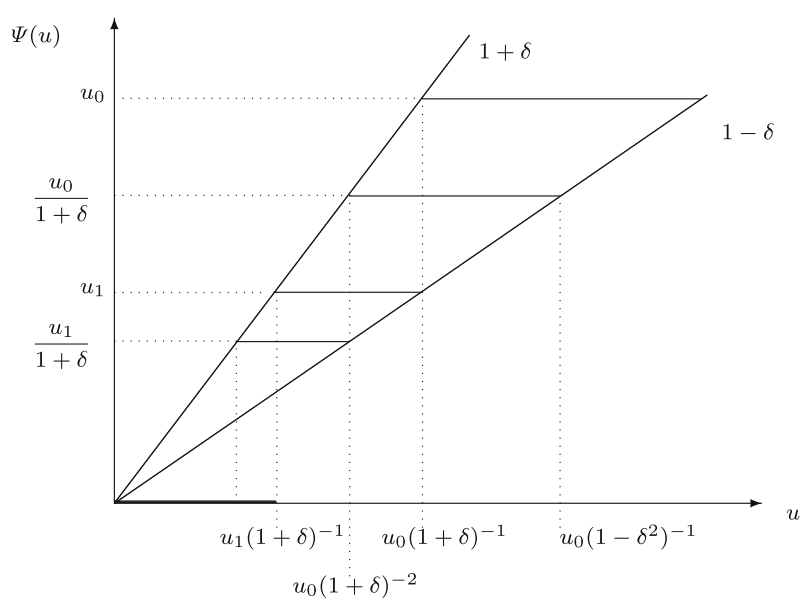

Fig. 1 The multi-valued map $\Psi(u)$ for $u>0$, and with $j=1$

quantization levels) are to be designed. Although it would be more correct to denote explicitly the dependence of $\Psi$ on $u_{0}, j$, i.e. to have $\Psi_{j, u_{0}}(u)$, this is not pursued in the paper to avoid cumbersome notations.

\subsection{Quantized time-delay systems}

We are interested in investigating the stability of the quantized time-delay system

$$
\dot{x}(t)=f(x(t))+g(x(t)) \Psi(u(t-\tau)),
$$

with $x(t) \in \mathbb{R}^{n}, n \geq 1, f(x), g(x)$ locally Lipschitz functions, and $\tau$ a positive real number, when $u(t)=z(x(t))$, with $z(\cdot)$ a continuously differentiable real-valued function to be designed. Since $\Psi(u(t-\tau))$ is a multi-valued function, we must specify the rule by which $\Psi(u(t-\tau))$ takes value in $U$ depending on its argument $u(t-\tau)$.

Consider the initial condition $\varphi \in C^{1}\left([-2 \tau, 0], \mathbb{R}^{n}\right)$ and let $T<\tau$ be a suitable positive number. For $t \in[0, T)$ we focus our attention on $\Psi(\bar{z}(t))$, where to ease the notation we have set $\bar{z}(t):=z(\varphi(t-\tau))$. At time $t=0$, depending on $|\bar{z}(0)|$, the value taken by the quantizer is specified as follows:

$$
\Psi(\bar{z}(0))=\left\{\begin{array}{lrl}
u_{i} \operatorname{sgn}(\bar{z}(0)), & \frac{1}{1+\delta} u_{i}<|\bar{z}(0)| \leq \frac{1}{1-\delta} u_{i}, 0 \leq i \leq j \\
0, & 0 \leq|\bar{z}(0)| \leq \frac{1}{1+\delta} u_{j} .
\end{array}\right.
$$

For all $t \in[0, T)$, we describe the law according to which $\Psi(\bar{z}(t))$ evolves as the argument $\bar{z}(t)$ varies. Before that, in order to have a concise description, we rename the quantization levels as follows:

$$
\tilde{u}_{k}:= \begin{cases}u_{k / 2} & k \text { even } \\ \frac{u_{(k-1) / 2}}{1+\delta} & k \text { odd }, \quad k=0,1, \ldots, 2 j+1,\end{cases}
$$



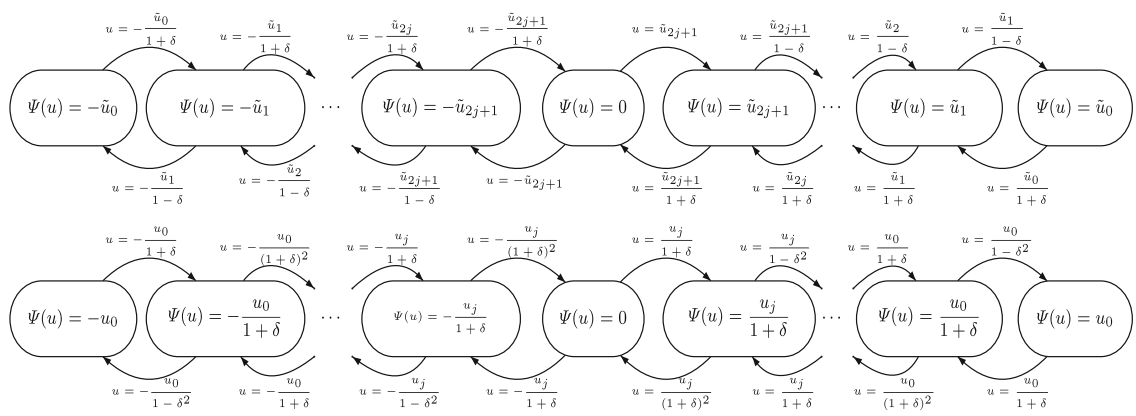

Fig. 2 The graph at the top illustrates the law (7) which describes the evolution of $\Psi(u(t))$ as $u(t)=\bar{z}(t)$ varies. Each edge connects two nodes, and is labeled with the condition which triggers the transition from the starting node to the destination node. The graph at the bottom illustrates the same law but with nodes and edges now labeled making use of the original values $u$ rather than $\tilde{u}$

and moreover we set $\tilde{u}_{2 j+2}:=0$. The evolution of $\Psi(\bar{z}(t))$ obeys the law below (a pictorial representation of the law is given by the directed graph in Fig. 2), where the symbol $\wedge$ denotes the logical conjunction 'and':

$$
\begin{aligned}
& |\Psi(\bar{z}(t))|=\tilde{u}_{k} \wedge|\bar{z}(t)|=\frac{\tilde{u}_{k}}{1+\delta} \Rightarrow\left|\Psi\left(\bar{z}\left(t^{+}\right)\right)\right|=\tilde{u}_{k+1}, \quad \text { for } k=0,1, \ldots, 2 j+1 \\
& |\Psi(\bar{z}(t))|=\tilde{u}_{k} \wedge|\bar{z}(t)|=\frac{\tilde{u}_{k}}{1-\delta} \Rightarrow\left|\Psi\left(\bar{z}\left(t^{+}\right)\right)\right|=\tilde{u}_{k-1}, \quad \text { for } k=1,2, \ldots, 2 j+1 \\
& |\Psi(\bar{z}(t))|=\tilde{u}_{k} \wedge|\bar{z}(t)|=\tilde{u}_{k-1} \Rightarrow\left|\Psi\left(\bar{z}\left(t^{+}\right)\right)\right|=\tilde{u}_{k-1}, \quad \text { for } k=2 j+2 .
\end{aligned}
$$

If none of the conditions on the right-hand side of the implications above is satisfied, then $\Psi\left(\bar{z}\left(t^{+}\right)\right)=\Psi(\bar{z}(t))$. Observe that (7) takes into account both the positive and the negative values of $\Psi(\bar{z}(t))$. In fact, since $\Psi(u) u \geq 0$ for all $u$, if $\Psi(\bar{z}(t))>0$ (respectively, $\Psi(\bar{z}(t))<0)$ so is $\bar{z}(t)$ and $\Psi\left(\bar{z}\left(t^{+}\right)\right)$. Hence, (7) is in good accordance with Fig. 2.

We now specify the solution we adopt for the system

$$
\dot{x}(t)=f(x(t))+g(x(t)) \Psi(\bar{z}(t))
$$

with $t \in[0, T)$. Set $t_{0}=0$, let $\Psi\left(\bar{z}\left(t_{0}\right)\right)$ be as in (6), compute $\Psi\left(\bar{z}\left(t_{0}^{+}\right)\right)$according to (7) above, and consider the solution $x(t)$ of

$$
\dot{x}(t)=f(x(t))+g(x(t)) \Psi\left(\bar{z}\left(t_{0}^{+}\right)\right)
$$

starting from the initial condition $x_{0}=\varphi(0)$, on the interval $\left[t_{0}, t_{1}\right]$, where $t_{1}$ is a time at which $\bar{z}(t)$ satisfies one of the conditions which force $\Psi(\bar{z}(t))$ to take a new value, provided that the solution of (9) can be extended up to $t_{1}$. By definition, $\Psi(\bar{z}(t))=\Psi\left(\bar{z}\left(t_{0}^{+}\right)\right)$for all $t \in\left[t_{0}, t_{1}\right]$, and on $\left[t_{0}, t_{1}\right], x(t)$ is equivalently the solution of (8). Then, set $x_{1}=x\left(t_{1}\right)$, compute $\Psi\left(\bar{z}\left(t_{1}^{+}\right)\right)$, and consider the solution of

$$
\dot{x}(t)=f(x(t))+g(x(t)) \Psi\left(\bar{z}\left(t_{1}^{+}\right)\right)
$$


starting from $x_{1}$, and defined on $\left[t_{1}, t_{2}\right]$, where $t_{2}$ is a time at which a new transition occurs. Iterating this argument, one finds a sequence $t_{0}, t_{1}, \ldots, t_{k}, t_{k+1}$ (for some integer $k \geq 0$, and where we have conventionally set $t_{k+1}=T$ ) of switching times, and the solution $x(t)$ of (8) on $[0, T)$ is a $\bar{C}^{1}$ function of time such that, for each $i=0,1, \ldots, k$, for all $t \in\left[t_{i}, t_{i+1}\right)$, it satisfies

$$
\dot{x}(t)=f(x(t))+g(x(t)) \Psi\left(\bar{z}\left(t_{i}^{+}\right)\right)
$$

Remark 1 We now explain why chattering is avoided in the interval $[0, T)$ thanks to the introduction of additional levels in the quantizer (see also [5]). In the proof of the main result below it is shown that this property is true for all the times. As a matter of fact, by the definition of (1), each time $\Psi(\bar{z}(t))$ makes a transition from one value to another, some (dwell) time will elapse before a new transition can occur. ${ }^{1}$ This can be illustrated with the help of Fig. 1, where $u$ is replaced by $\bar{z}(t)$. Suppose that, at time $t, \Psi(\bar{z}(t))=u_{0}$ and $\bar{z}(t)$ hits the point $\frac{u_{0}}{1+\delta}$. Then $\Psi(\bar{z}(t))$ takes the new value $\frac{u_{0}}{1+\delta}$ (see Fig. 1). After the switching, the function $\bar{z}(t)$ can increase and eventually hits the point $u_{0}\left(1-\delta^{2}\right)^{-1}$, or decrease and eventually hits the point $u_{0}(1+\delta)^{-2}$ (if it hits none of the two points then this means that $\bar{z}(t)$ remains in the interval $\left(u_{0}(1+\delta)^{-2}, u_{0}\left(1-\delta^{2}\right)^{-1}\right)$ for the entire interval $[t, T)$, and no switching occurs in this interval). In either case, before a new transition takes place, some time will elapse, because the function $\bar{z}(t)$ must cover an interval of finite length with finite speed. In fact, for a given initial condition $\varphi \in C^{1}\left([-2 \tau, 0], \mathbb{R}^{n}\right)$, with $\|\varphi\|_{c} \leq R$ and $R>0$, the time derivative of $\bar{z}(t)=z(\varphi(t-\tau))$ is continuous and bounded on [0,T), and in particular:

$$
\left|\frac{d \bar{z}(t)}{d t}\right| \leq \max _{|x| \leq R}\left|\frac{\partial z(x)}{\partial x}\right| \cdot \max _{t \in[-2 \tau,-\tau]}\left|\frac{d \varphi(t)}{d t}\right| .
$$

If, on the other hand, we were adopting the quantizer (4), $\Psi(\bar{z}(t))$ would have taken the value $u_{1}$ rather than $\frac{u_{0}}{1+\delta}$. Immediately after the switching, it could happen that $\bar{z}(t)$ cannot decrease, thus forcing a transition to the previous value, which would in turn trigger a new transition to $u_{1}$, and this would continue to happen again and again. It is precisely to avoid such fast transitions that new quantization levels were added. This addition can be seen as a way to add hysteresis to the quantized system, and we will refer to (1) as a quantizer with hysteresis.

For the analysis to follow, the following observation is important. For each $t \in[0, T)$, such that $t \in\left[t_{i}, t_{i+1}\right), i=0,1, \ldots, k$, if $|\bar{z}(t)|<u_{0}(1-\delta)^{-1}$, then the solution $x(t)$ of (8) satisfies the differential inclusion

$$
\dot{x}(t) \in f(x(t))+g(x(t)) K(\Psi(\bar{z}(t))),
$$

\footnotetext{
1 For some classes of nonlinear systems, it is possible to estimate a lower bound on such a dwell time [2]. This is particularly important in the case in which the quantized controller is implemented over a network, since it gives indications on the data-rate needed to transmit the quantized information.
} 
where $K(\Psi(u))$, with $u=\bar{z}(t)$, is such that

$$
K(\Psi(u)) \subseteq\left\{\begin{aligned}
\{v \in \mathbb{R}: v= & (1+\lambda \delta) u, \lambda \in[-1,1]\} \\
& (1+\delta)^{-1} u_{j}<|u| \leq(1-\delta)^{-1} u_{0} \\
\{v \in \mathbb{R}: v= & (1+\delta) u, \lambda \in[0,1]\},|u| \leq(1+\delta)^{-1} u_{j}
\end{aligned}\right.
$$

This is easily verified bearing in mind that, by the definition (1) of the map $\Psi(u)$, $\Psi(u) \in K(\Psi(u))$ for all $|u|<u_{0}(1-\delta)^{-1}$.

\subsection{Problem formulation}

Since the control action is zero in the vicinity of the origin due to the dead-zone of the quantizer $\left(\Psi(u)=0\right.$ for $\left.|u| \leq u_{j}(1+\delta)^{-1}\right)$, asymptotic stability of the origin of (5) is not possible to achieve (except in exceptional cases without interest). We are rather interested in the following property:

Definition 1 The system

$$
\dot{x}(t)=f(x(t))+g(x(t)) v(t-\tau),
$$

with $\tau \geq 0$ is semi-globally practically stabilizable by quantized feedback if for any $\varepsilon<R<0$ there exist a law $z(x)$, a real number $u_{0}>0$ and an integer $j \geq 1$ such that the solution of

$$
\dot{x}(t)=f(x(t))+g(x(t)) \Psi(z(x(t-\tau))),
$$

starting from $\mathcal{R}=\left\{\varphi \in C^{1}\left([-2 \tau, 0], \mathbb{R}^{n}\right):\|\varphi\|_{c} \leq R\right\}$ enters $B_{\varepsilon}$, the closed ball of radius $\varepsilon$, at some finite time $t_{s} \geq 0$, and remains in that set for all $t \geq t_{s}$.

In the remaining sections, we propose a solution to the problem formulated above.

Remark 2 The difficulty to achieve asymptotic stability can be seen by rewriting the system (14) in the form of a nominal stable system affected by a perturbation, namely

$$
\dot{x}(t)=f(x(t))+g(x(t)) z(x(t))+g(x(t))[\Psi(z(x(t-\tau)))-z(x(t))],
$$

and neglecting the effect of the delay (the presence of the delay worsens the situation). Consider the situation in which $z(x(t))$, the quantity which undergoes quantization, is close to zero, namely $|z(x(t))|<(1+\delta)^{-1} u_{j}$. Bearing in mind (12), the perturbation $|\Psi(z(x(t)))-z(x(t))|$ is bounded from above by $|\lambda(\delta+1)-1||z(x(t))|$, with $\lambda \in[0,1]$ (the argument will be made clearer later on). Even in the easy case in which the system is exponentially stable, asymptotic stability cannot be proven unless the perturbation (in this case $[\lambda(\delta+1)-1] z(x(t)))$ is bounded by a linear term $\gamma|x(t)|$ and $\gamma$ is sufficiently small (see e.g. [10, Sect. 5.1]), conditions which are not met in our scenario. For the majority of the systems, these conditions are not satisfied either and other notions of stability have been introduced. A notion of stability for solutions 
of systems affected by non-vanishing perturbations is that of uniform ultimate boundedness $[6,11]$ which has found wide application in the area of robust control (see, e.g. [10]). The notion of semi-global practical stabilizability we consider in our paper has been extensively investigated for problems of robust stabilization of nonlinear systems (see, e.g. [7, Chapter 12], [21], and references therein). The same notion of stability has been already studied for quantized time-delay systems as well [13,20].

\section{Standing assumptions and main result}

\subsection{Basic assumptions}

The result to be derived below for the system (13) holds under the following standing assumptions.

(A1) There exist a continuously differentiable positive definite and proper Lyapunov function $V(x)$, two class $\mathcal{K}_{\infty}$ functions $\kappa_{1}, \kappa_{2}$, a positive definite continuous function $W(x)$ and a continuously differentiable real-valued function $z(x)$, which is zero at the origin, with $W(x)$ and $z(x)$ both depending on $\delta$, such that, for all $x \in \mathbb{R}^{n}$,

$$
\begin{aligned}
& \kappa_{1}(|x|) \leq V(x) \leq \kappa_{2}(|x|), \\
& \frac{\partial V}{\partial x}[f(x)+g(x)(1+p) z(x)] \leq-W(x), p \in[-\delta, \delta] .
\end{aligned}
$$

Remark 3 It would be slightly more correct to denote $W(x)$ and $z(x)$ by, respectively, $W_{\delta}(x)$ and $z_{\delta}(x)$, since due to the presence of the uncertainty in the input channel, both these functions are going to depend on the size $\delta$ of the uncertainty (see Sect. 3.2 below). However, to ease the notation, we decided not to make the dependence on $\delta$ explicit.

Remark 4 The uncertainty in the input channel is modeled through the parameter $p$, whose range depends on the quantization density through $\delta$. Such uncertainty takes into account the effect due to quantization, as it should be evident from (12). Assumption (A1) amounts to require the system $\dot{x}(t)=f(x(t))+g(x(t)) u(t)$, with no delay, to be stabilizable in the presence of quantization. The design of a stabilizing quantized feedback is carried out, e.g. in [1] (see also Sect. 3.2 below).

The next two assumptions require the system to be robust with respect to delays. In particular they are needed to guarantee that no finite-escape time phenomenon will occur, and that the solution stays bounded for all the times. These conditions also appear in [14] (where no quantization was present), although in a slightly different form. The difference is due to the fact that the quantization effect adds up to the delay effect, and in the conditions below also the quantization parameter $\delta$ plays a role. More comments on these two assumptions are postponed to Section 3.3.

(A2) Let $\Omega$ be a positive real number which satisfies $\Omega \geq 16 \tau$. For all $x \in \mathbb{R}^{n}$, for all $\xi \in \bar{C}^{1}\left([0,2 \tau], \mathbb{R}^{n}\right)$, for all $\lambda_{1} \in[-1,1]$ and for all $\lambda_{2} \in \bar{C}^{0}([0, \tau], \mathbb{R})$ 
such that $\lambda_{2}(m) \in[1-\delta, 1+\delta]$ for all $m \in[0, \tau]$, the inequality

$$
-\frac{1}{4} W(x)-T\left(x, \xi, \lambda_{1}, \lambda_{2}\right)-\frac{1}{\Omega} \int_{0}^{2 \tau} W(\xi(\ell)) \mathrm{d} \ell \leq 0,
$$

with

$$
\begin{aligned}
T\left(x, \xi, \lambda_{1}, \lambda_{2}\right) & =L_{g} V(x)\left(1+\lambda_{1} \delta\right) \int_{\tau}^{2 \tau} H\left(\xi(\ell), \xi(\ell-\tau), \lambda_{2}(\ell-\tau)\right) \mathrm{d} \ell \\
H(a, b, c) & =L_{f} z(a)+L_{g} z(a) c z(b)
\end{aligned}
$$

holds.

(A3) There exists a nondecreasing function $\kappa_{3}(\cdot)$ of class $C^{1}$ such that for all $x \in \mathbb{R}^{n}$, for all $L \geq 0$ and for all $\lambda \in[-1,1]$, the inequality

$$
-\frac{1}{2} W(x)+\sup _{|a| \leq L}\left\{L_{g} V(x)(1+\lambda)[z(a)-z(x)]\right\} \leq \kappa_{3}(L)[V(x)+1]
$$

holds. Let $\kappa_{4}(L)=2 \kappa_{3}(L)$.

The two subsections below provide comments to help the readers to understand the role played by each assumption in the solution of the problem. However, the reader who is interested in getting to the statement of the main result immediately, can skip the next two subsections and go directly to Sect. 3.4.

\subsection{Comments on the Assumption (A1)}

A number of ways to have Assumption (A1) fulfilled are discussed below.

- Lyapunov Redesign. Suppose that, for the system (13), are known a function $V$ of class $C^{2}$, and a function $\zeta(x)$ of class $C^{1}$ such that, instead of (15), only the weaker condition

$$
L_{f} V(x)+L_{g} V(x) \zeta(x)=-\tilde{W}(x),
$$

with $\tilde{W}(x)$ a continuous positive definite function, is satisfied. Introduce the control law

$$
z(x)=\zeta(x)-\alpha(x) L_{g} V(x),
$$


with $\alpha(x)$ a positive function to be chosen later. Then we have

$$
\begin{aligned}
\frac{\partial V}{\partial x} & {[f(x)+g(x)(1+p) z(x)] } \\
= & \frac{\partial V}{\partial x}[f(x)+g(x) \zeta(x)]-\alpha(x)\left|L_{g} V(x)\right|^{2} \\
& +p L_{g} V(x)\left[\zeta(x)-\alpha(x) L_{g} V(x)\right] \\
\leq & -W(x)-\alpha(x)(1+p)\left|L_{g} V(x)\right|^{2}+p L_{g} V(x) \zeta(x) \\
\leq & -W(x)-\alpha(x)(1-\delta)\left|L_{g} V(x)\right|^{2}+\delta\left|L_{g} V(x)\right||\zeta(x)| .
\end{aligned}
$$

A simple completion-of-the-squares argument shows that

$$
\frac{\partial V}{\partial x}[f(x)+g(x)(1+p) z(x)] \leq-\frac{3}{4} \tilde{W}(x),
$$

provided that

$$
\alpha(x) \geq \frac{\delta^{2}}{1-\delta} \frac{|\zeta(x)|^{2}}{\tilde{W}(x)} .
$$

Hence, the control law (19), with $\alpha(x)$ defined above and such that $\lim _{x \rightarrow 0}$ $\alpha(x) L_{g} V(x)=0$, guarantees the fulfillment of Assumption (A1) with $W(x)=3 \tilde{W}(x) / 4$.

- Sontag's universal stabilizer [19]. Consider the system

$$
\dot{x}=f(x)+g(x)[1+p] u,
$$

with $x \in \mathbb{R}^{n}, u \in \mathbb{R}, p \in[-\delta, \delta], \delta \in[0,1)$. Let us assume that a control Lyapunov function $V(x)$ is known for the system (21) with $p=0$, and set

$$
\dot{V}(x)=a(x)+[1+p] b(x) u,
$$

with

$$
a(x)=L_{f} V(x), b(x)=L_{g} V(x) .
$$

Since $V$ is a control Lyapunov function for (21) with $p=0, b(x)=0$ implies $a(x)<0$ when $x \neq 0$. Next, consider the control given by Sontag's formula:

$$
\begin{aligned}
& u(x)=K \frac{-a(x)-\sqrt{a(x)^{2}+b(x)^{4}}}{b(x)} \text { when } b(x) \neq 0, \\
& u(x)=0 \quad \text { when } b(x)=0,
\end{aligned}
$$

and where $K$ is a positive real number to be selected later. Then, when $b(x) \neq 0$, the derivative of $V$ along the trajectories of (21) in closed-loop with $u(x)$ defined 
in (24) satisfies

$$
\begin{aligned}
\dot{V}(x) & =a(x)+[1+p] b(x) K \frac{-a(x)-\sqrt{a(x)^{2}+b(x)^{4}}}{b(x)} \\
& =a(x)-[1+p] K a(x)-[1+p] K \sqrt{a(x)^{2}+b(x)^{4}} \\
& =[1-(1+p) K] a(x)-[1+p] K \sqrt{a(x)^{2}+b(x)^{4}} .
\end{aligned}
$$

We choose $K=\frac{2}{1-\delta}>0$. Then, when $a(x) \geq 0$, we have

$$
\dot{V}(x) \leq-a(x)-[1+p] K \sqrt{a(x)^{2}+b(x)^{4}},
$$

and, when $a(x)<0$,

$$
\dot{V}(x)=a(x)-[1+p] K\left(a(x)+\sqrt{a(x)^{2}+b(x)^{4}}\right)<0 .
$$

When $b(x)=0$, then

$$
\dot{V}(x)=a(x)<0 \text { if } x \neq 0 .
$$

Under the small control property [19] one can prove that the control law introduced above is smooth everywhere except at the origin where it may be only continuous. However, in many cases, the control law turns out to be also continuously differentiable at the origin, and then a continuously differentiable function $z(x)$ which guarantees the inequality (15) is obtained.

- Lyapunov stable systems. Consider again system (21), and assume that a Lyapunov function $V(x)$ such that $a(x) \leq 0$, and $b(x) \neq 0$ when $x \neq 0$ and $a(x)=0$, is known. Then, selecting

$$
u=-\xi(x) b(x),
$$

where $\xi$ is any $C^{1}$ positive function, we obtain, for all $x \in \mathbb{R}^{n}$

$$
\dot{V}(x) \leq a(x)-[1-\delta] \xi(x) b(x)^{2}
$$

and the function $a(x)-[1-\delta] \xi(x) b(x)^{2}$ is negative definite. Inequality (15) then holds with $z(x)=-\xi(x) b(x)$ and $W(x)=-a(x)+[1-\delta] \xi(x) b(x)^{2}$.

- Dissipation inequality [1,5]. Consider the system (13). Suppose that a Lyapunov function $V(x)$ is known such that for all $x \in \mathbb{R}^{n}$

$$
L_{f} V(x)-\frac{1}{4}\left(1-\delta^{2}\right)\left(L_{g} V(x)\right)^{2} \leq-\tilde{W}(x)
$$

Then, for any $p \in[-\delta, \delta]$ it is also true that

$$
L_{f} V(x)-\frac{1}{4}\left(1-p^{2}\right)\left(L_{g} V(x)\right)^{2} \leq-\tilde{W}(x) .
$$


Define now

$$
z(x)=-\frac{1}{2} L_{g} V(x)
$$

and observe that the inequality above rewrites as

$$
L_{f} V(x)+\frac{1}{4} p^{2}\left(L_{g} V(x)\right)^{2}+z(x) L_{g} V(x)+z(x)^{2} \leq-\tilde{W}(x),
$$

or, equivalently,

$$
\frac{\partial V}{\partial x}(f(x)+g(x) z(x))+\frac{1}{4} p^{2}\left(L_{g} V(x)\right)^{2}+z(x)^{2} \leq-\tilde{W}(x) .
$$

We remark incidentally [1] that the latter inequality implies the existence of a control $u=z(x)$ which renders the system

$$
\left\{\begin{array}{l}
\dot{x}=f(x)+g(x) u+g(x) w \\
z=u
\end{array}\right.
$$

strictly dissipative with respect to the supply rate $q(w, z)=-z^{2}+p^{-2} w^{2}$.

Observe now that

$$
p z(x) L_{g} V(x) \leq \frac{1}{4} p^{2}\left(L_{g} V(x)\right)^{2}+z(x)^{2}
$$

and therefore (31) implies that

$$
\frac{\partial V}{\partial x}(f(x)+g(x) z(x))+p z(x) L_{g} V(x) \leq-\tilde{W}(x),
$$

that is (15) with $W(x)=\tilde{W}(x)$.

\subsection{Comments on the Assumptions (A2) and (A3)}

The two Assumptions (A2) and (A3) describe, in terms of the Lyapunov function $V$, how robust with respect to delays in the input channel the system $\dot{x}(t)=f(x(t))+$ $g(x(t)) u(t)$ should be in order to find a stabilizing feedback despite the delay. The role of these assumptions for systems with no quantization was already investigated in [14]. To better assess such a role, let us neglect the effect due to the quantization, and let us set $\delta=0$. Then, in Assumption (A1), $p=0$ and (15) becomes a standard stabilizability assumption. The inequality (16) in (A2) becomes

$$
-\frac{1}{4} W(x)-T(x, \xi)-\frac{1}{\Omega} \int_{0}^{2 \tau} W(\xi(\ell)) \mathrm{d} \ell \leq 0,
$$


with

$$
\begin{aligned}
& T(x, \xi)=L_{g} V(x) \int_{\tau}^{2 \tau} H(\xi(\ell), \xi(\ell-\tau)) \mathrm{d} \ell, \\
& H(a, b)=L_{f} z(a)+L_{g} z(a) z(b) .
\end{aligned}
$$

Similarly, in (17), $\lambda=0$, and the inequality implies that for all $\xi \in \mathcal{C}^{1}\left([0,2 \tau], \mathbb{R}^{n}\right)$, there exists a positive constant $\kappa \xi$ such that, for all $x \in \mathbb{R}^{n}$, for all $t \in[0,2 \tau]$,

$$
-\frac{1}{2} W(x)+L_{g} V(x)[z(\xi(t))-z(x)] \leq \kappa \xi[V(x)+1] .
$$

The conditions (32), (33) coincide with those found in [14] to prove that the origin of

$$
\dot{x}(t)=f(x(t))+g(x(t)) z(x(t-\tau))
$$

is uniformly globally asymptotically stable. Compared with [14], the stronger conditions we have in this paper are due to the fact that both quantization and delay affect the system.

In the case no quantization is present, the role of (32), (33) to guarantee stability of time-delay systems is easier to describe (see [14] for details). The condition (33), for instance, guarantees that no finite-time escape of the solution occurs. As a matter of fact, the time derivative of $V$ computed along the solutions of (34) obeys the equations

$$
\begin{aligned}
\dot{V}(x(t)) & =L_{f} V(x(t))+L_{g} V(x(t)) z(x(t-\tau)) \\
& =L_{f} V(x(t))+L_{g} V(x(t)) z(x(t))+L_{g} V(x(t))[z(x(t-\tau))-z(x(t))] \\
& \leq-W(x(t))+L_{g} V(x(t))[z(x(t-\tau))-z(x(t))] .
\end{aligned}
$$

As $t$ ranges in the interval [0, $\tau], x(t-\tau)$ can be viewed as a function $\xi \in \mathcal{C}^{1}([-\tau, 0]$, $\left.\mathbb{R}^{n}\right)$, and bearing in mind (33), we have $\dot{V}(x(t)) \leq K_{\xi}(V(x(t))+1)$. From this we infer that no finite escape-time can exist on $\left[t_{0}, t_{0}+\tau\right]$. Iterating the argument, one can prove that the solution is defined for all $t$.

The condition (32) guarantees that a suitable Lyapunov-Krasowskii functional is strictly decreasing along the solutions of the closed-loop system (again, the interested reader is referred to [14] for more details). The purpose of the rest of the paper is to show how, taking advantage of Assumptions (A1)-(A3), the arguments of [14] can be modified to take into account the additional constraints due to the presence of the quantizer.

We stress that the conditions (32), (33) require the system to be robust with respect to (quantization and) delays and are essential to design stabilizing control laws for nonlinear (quantized) time-delay systems. Analogous conditions are found in other contributions on the topic. In [20], using an approach based on Razumikhin-like theorems, uniform asymptotic stability with restriction $\Delta$ on the norm $\|\varphi\|_{c}$ of the initial condition and with offset $\varepsilon$ (a notion of stability very similar to what we have in Definition 1) is proven. To be more precise, suppose the system is stabilizable, that is (A1) 
holds (with $p=\delta=0$ ). Also suppose for the sake of simplicity that $W(x)$ is replaced by the class- $\mathcal{K}_{\infty}$ function $\alpha_{3}(|x|)$. Then it is possible to design a smooth invertible function $G(x)$ and a class- $\mathcal{K}_{\infty}$ function $\gamma_{\theta}$ such that

$$
|x(t)| \geq \gamma_{\theta}(|\theta(t)|) \Rightarrow \dot{V}(t) \leq-\frac{1}{2} \alpha_{3}(|x(t)|),
$$

with

$$
\begin{aligned}
\theta(t) & =-G^{-1}(x(t))[z(x(t))-z(x(t-\tau))] \\
& =-\left.G^{-1}(x(t)) \int_{t-\tau}^{t} \frac{\partial z(x)}{\partial x}\right|_{x=x(s)}[f(x(s))+g(x(s)) z(x(s-\tau))] \mathrm{d} s .
\end{aligned}
$$

Further, one can find a class- $\mathcal{K}$ function $\gamma_{1}$ such that

$$
|\theta(t)| \leq \tau \gamma_{1}\left(\sup _{t-2 \tau \leq s \leq t}|x(s)|\right) .
$$

Hence, combining (35) and (36), one obtains:

$$
|x(t)| \geq \gamma_{\theta}\left(\tau \gamma_{1}\left(\sup _{t-2 \tau \leq s \leq t}|x(s)|\right)\right) \Rightarrow \dot{V}(t) \leq-\frac{1}{2} \alpha_{3}(|x(t)|) .
$$

Under the small-gain condition

$$
\kappa_{1}^{-1} \circ \kappa_{2} \circ \gamma_{\theta}\left(\tau \gamma_{1}(s)\right)<s, \text { for all } \varepsilon<s<\Delta,
$$

the inequality above shows that the zero solution of the system

$$
\dot{x}(t)=f(x(t))+g(x(t)) \zeta(x(t-\tau))
$$

is uniformly asymptotically stable with restriction $\Delta$ on the norm $\|\varphi\|_{c}$ of the initial condition, and with offset $\delta .^{2}$

The condition (37) represents an alternative way to express robustness of the system with respect to delays to infer stability results using Razumikhin-like theorems.

\subsection{Main result}

We are ready to state the main result of our work. As already made clear in the problem formulation (Definition 1), the two main design parameters are the range $u_{0}$ and the number $j$ of levels of the quantizer. Intuitively, to design $u_{0}$ we need to quantify the

\footnotetext{
2 In the terminology of [21], the offset is the size of the set where the state converges at some finite time and stays there from that time on-in our paper such a parameter is denoted by $\varepsilon$.
} 
"overshoot" of the state variable and we expect this to depend on the size of the initial condition. Regarding the number of quantization levels $j$, it is not hard to figure out that in general the closer one wants to confine the state to the origin (i.e. the smaller $\varepsilon$ is in Definition 1), the larger the number of quantization levels must be. On the other hand, having fixed the width of the quantizer, the number of the quantization levels will increase with the range $u_{0}$ and in turn with $R$. Such a dependence is made clear in the statement below. The proof is constructive and provides the explicit expressions for $u_{0}$ and $j$.

Proposition 1 Let us assume that the system (13) satisfies Assumptions (A1)-(A3). Then the origin of (13) is semi-globally practically stabilizable by quantized feedback. Namely, there exist a positive, continuous and non-decreasing function $u_{0}(\cdot)$ : $\mathbb{R}_{\geq 0} \rightarrow \mathbb{R}_{>0}$, and a positive continuous function $j(\cdot, \cdot): \mathbb{R}_{\geq 0}^{2} \rightarrow \mathbb{R}_{>0}$ such that, for any $R>\varepsilon>0$, if $u_{0} \geq u_{0}(R), j \geq j(\varepsilon, R)$ and $z$ is the feedback provided by Assumption (Al) satisfying (15), then the solution of (14) starting from $\mathcal{R}=\{\varphi \in$ $\left.C^{1}\left([-2 \tau, 0], \mathbb{R}^{n}\right):\|\varphi\|_{c} \leq R\right\}$ enters $B_{\varepsilon}$, the closed ball of radius $\varepsilon$, at some finite time $t_{s} \geq 0$, and remains in that set for all $t \geq t_{s}$.

The proof of the result is postponed to the next section. Before ending Sect. 3, we discuss two examples in which the proposition above is applied.

\subsection{Example 1}

We illustrate Proposition 1 by showing how it applies when the functions $f$ and $g$ in (13) are linear. Thus, we consider the system

$$
\dot{x}(t)=A x(t)+B \Psi(u(t-\tau))
$$

where $A \in \mathbb{R}^{n \times n}$ and $B \in \mathbb{R}^{n \times 1}$ are constant matrices. We assume that the pair $(A, B)$ be stabilizable. Then there exist a positive definite symmetric matrix $Q \in \mathbb{R}^{n \times n}$ and a matrix $\tilde{K} \in \mathbb{R}^{1 \times n}$ such that

$$
(A+B \tilde{K})^{\top} Q+Q(A+B \tilde{K})=-I
$$

where $I \in \mathbb{R}^{n \times n}$ denotes the identity matrix. Then, in view of the Lyapunov redesign we have proposed to determine a control law such that Assumption (A1) is verified, one can verify that the matrix

$$
K=\tilde{K}-2 \alpha Q B, \quad \text { with } \quad \alpha \geq \frac{\delta^{2}}{1-\delta}|K|^{2},
$$

is such that, for all $x \in \mathbb{R}^{n}$,

$$
2 x^{\top} Q[A x+B(1+p) K x] \leq-c x^{T} x, \quad p \in[-\delta, \delta]
$$


with $c=3 / 4$. Therefore, Assumption (A1) is satisfied with $V(x)=x^{\top} Q x$, $z(x)=K x$, and $W(x)=c x^{\top} x$. Hence in what follows we let $Q, K, c$ be such that (39) holds.

We turn now to Assumption (A2). We have

$$
\begin{aligned}
-T\left(x, \xi, \lambda_{1}, \lambda_{2}\right)= & -2\left(1+\lambda_{1} \delta\right) x^{T} Q B \int_{\tau}^{2 \tau} K\left\{A \xi(l)-B \lambda_{2}(\ell-\tau) K \xi(\ell-\tau)\right\} \mathrm{d} \ell \\
\leq & 2(1+\delta)|x||Q B| \int_{\tau}^{2 \tau}|K A||\xi(\ell)| \mathrm{d} \ell \\
& +2(1+\delta)^{2}|x||Q B| \int_{\tau}^{2 \tau}|K B K||\xi(\ell-\tau)| \mathrm{d} \ell \\
\leq & 2(1+\delta)|x||Q B| \int_{\tau}^{2 \tau}\left[G_{1}|\xi(\ell)|+G_{2}|\xi(\ell-\tau)|\right] \mathrm{d} \ell
\end{aligned}
$$

with $G_{1}=|K A|, G_{2}=(1+\delta)|K B K|$. In view of the bounds on $T\left(x, \xi, \lambda_{1}, \lambda_{2}\right)$, Assumption (A2) is verified if

$$
-\frac{c}{4}|x|^{2}+2(1+\delta)|x||Q B| \int_{\tau}^{2 \tau}\left[G_{1}|\xi(\ell)|+G_{2}|\xi(\ell-\tau)|\right] \mathrm{d} \ell-\frac{c}{\Omega} \int_{0}^{2 \tau}|\xi(\ell)|^{2} \mathrm{~d} \ell \leq 0
$$

with $\Omega=16 \tau$. We easily deduce that (40) is satisfied if

$$
-\frac{c}{4}|x|^{2}+2 G_{3}|x| \int_{0}^{2 \tau}|\xi(\ell)| \mathrm{d} \ell-\frac{c}{16 \tau} \int_{0}^{2 \tau}|\xi(\ell)|^{2} \mathrm{~d} \ell \leq 0
$$

with $G_{3}=2|Q B|(1+\delta) \max \left\{G_{1}, G_{2}\right\}$. By Young's inequality applied to the second term, we deduce that (41) is satisfied if there exists $\varepsilon>0$ such that

$$
\left(-\frac{c}{4}+\frac{G_{3}^{2}}{\varepsilon}\right)|x|^{2}+\left(\varepsilon \cdot 2 \tau-\frac{c}{16 \tau}\right) \int_{0}^{2 \tau}|\xi(\ell)|^{2} \mathrm{~d} \ell \leq 0
$$

The inequality holds if $\varepsilon=4 G_{3}^{2} / c$ and

$$
\tau \leq \frac{c}{8 \sqrt{2} G_{3}} \leq \frac{c}{16(1+\delta) \sqrt{2}|Q B| \max \{|K A|,(1+\delta)|K B K|\}}
$$


Finally we consider Assumption (A3). The left-hand side of (17) becomes

$$
\begin{aligned}
- & \frac{1}{2} W(x)+\sup _{|a| \leq L}\left\{L_{g} V(x)(1+\lambda)[z(a)-z(x)]\right\} \\
& =-\frac{c}{2} x^{\mathrm{T}} x+\sup _{|a| \leq L}\left\{2 x^{\mathrm{T}} Q B(1+\lambda) K[a-x]\right\} \\
& \leq 4|Q B K||x|[|x|+L] \\
& \leq 4|Q B K|\left[(L+1)|x|^{2}+L\right] \\
& \leq 4|Q B K|(L+1)\left[\lambda_{\text {min }}^{-1}(Q) V(x)+1\right] .
\end{aligned}
$$

We deduce that one can find a constant $\Gamma=4|Q B K| \max \left\{\lambda_{\min }^{-1}(Q), 1\right\}$ such that Assumption (A3) is satisfied with $\kappa_{3}(\ell)=\Gamma(\ell+1)$. Summarizing, Assumptions (A1)-(A3) are satisfied for the system (38). Hence, we can conclude that Proposition 1 applies, provided that the pair $(A, B)$ is stabilizable, and the delay $\tau$ satisfies (43).

\subsection{Example 2}

In this section, we consider the classical equations of an actuated pendulum without friction:

$$
\begin{aligned}
& \dot{x}_{1}=x_{2} \\
& \dot{x}_{2}=-\sin x_{1}+u .
\end{aligned}
$$

The control law

$$
u=\zeta(x)=\sin x_{1}-x_{1}-2 x_{2}
$$

and the Lyapunov function

$$
V(x)=x^{\mathrm{T}} Q x=x^{\mathrm{T}}\left(\begin{array}{cc}
\frac{3}{2} & \frac{1}{2} \\
\frac{1}{2} & \frac{1}{2}
\end{array}\right) x
$$

are such that

$$
\frac{\partial V}{\partial x}(f(x)+g(x) \zeta(x))=-|x|^{2} .
$$

Applying the Lyapunov redesign of Sect. 3.2, it is straightforward to see that

$$
z(x)=\sin x_{1}-(\alpha+1) x_{1}-(\alpha+2) x_{2},
$$


with $\alpha \geq 16 \frac{\delta^{2}}{1-\delta}$, guarantees Assumption (A1) with $W(x)=\frac{3}{4}|x|^{2}$. To check Assumption (A2), observe that

$$
\begin{aligned}
& -T\left(x, \xi, \lambda_{1}, \lambda_{2}\right) \\
& \leq 2(1+\delta)|x| \int_{\tau}^{2 \tau}[2(2+\alpha)|\xi(\ell)|+(2+\alpha)(1+\delta) 2(2+\alpha)|\xi(\ell-\tau)|] \mathrm{d} \ell \\
& \leq 2(1+\delta)|x| 2(2+\alpha) \int_{\tau}^{2 \tau}[|\xi(\ell)|+(2+\alpha)(1+\delta)|\xi(\ell-\tau)|] \mathrm{d} \ell .
\end{aligned}
$$

Similarly to the previous example, one can prove that, if

$$
\tau \leq \frac{3}{128 \sqrt{2}(\alpha+2)^{2}(1+\delta)},
$$

then Assumption (A2) is fulfilled. Even Assumption (A3) can be easily verified. As a matter of fact,

$$
\begin{aligned}
& -\frac{1}{2} W(x)+\sup _{|a| \leq L}\left\{L_{g} V(x)(1+\lambda)[z(a)-z(x)]\right\} \\
& \quad \leq \sup _{|a| \leq L}\{2|x|(1+\delta) 2(\alpha+2)[|a|+|x|]\} \\
& \quad \leq 4(1+\delta)(\alpha+2)|x|[|x|+L] .
\end{aligned}
$$

As in the previous example, one can deduce that Assumption (A3) is fulfilled with $\kappa_{3}(\ell)=\Gamma(\ell+1)$ and $\Gamma=4(1+\delta)(\alpha+2) \frac{\sqrt{2}}{\sqrt{2}-1}$. The region under the graph in Fig. 3 describes the pairs $(\delta, \tilde{\tau})$, with $\tilde{\tau}=\frac{1}{(\alpha+2)^{2}(1+\delta)}$, for which the system (44) is semi-globally practically stabilizable.

\section{Proof of Proposition 1}

The proof is based on a Lyapunov-Krasowskii functional given by the sum of the Lyapunov function $V(x)$ in Assumption (A1) and a term which at time $t$ depends on the state $x(\cdot)$ restricted to the interval $[t-2 \tau, t]$. Hence, in order to use such a Lyapunov-Krasowskii functional, we need to first prove that all solutions of the closedloop system we consider exist for all $t \in[-2 \tau, 2 \tau]$. To this purpose, we will only make use of the Lyapunov function $V(x)$. Then we will prove that the solutions can be extended beyond $2 \tau$, showing that the Lyapunov-Krasowskii functional is bounded for all the time and finally that the solutions converge in finite time to a ball around the origin of radius $\varepsilon$. 


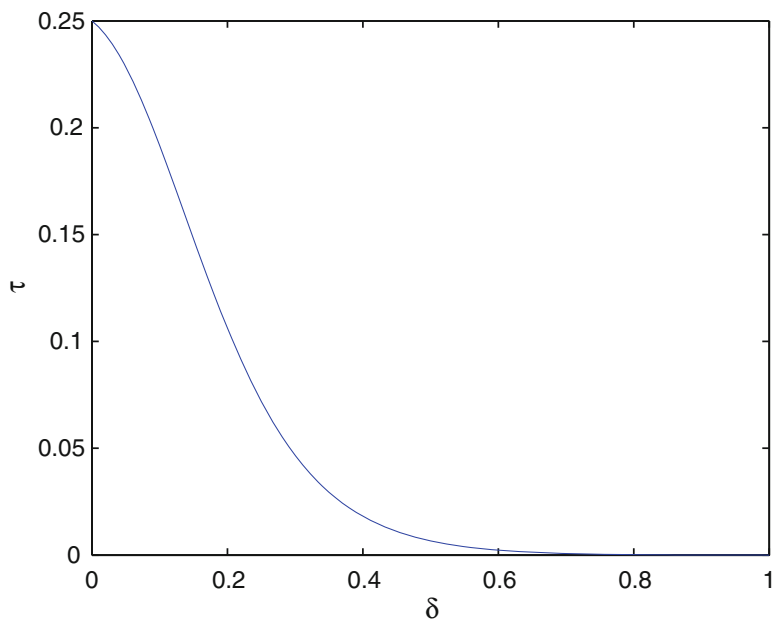

Fig. 3 The region under the graph represents the set of pairs $(\delta, \tilde{\tau})$ for which the system (44) is semi-globally practically stabilizable. In the picture, $\tilde{\tau}$ is simply denoted as $\tau$. As $\delta$ tends to 0 (no quantization) the normalized maximal allowable delay $\tilde{\tau}$ approaches its maximum

\subsection{Existence of solutions for $t \in[-2 \tau, 2 \tau]$}

As a first step, we need to define the function $u_{0}(\cdot)$ by which we define the range $u_{0}$. We have already observed that to find such a function, we need to estimate the region where the state is confined for all the times. We will obtain such an estimate by steps, first estimating a bound on $|x(t)|$ on the interval $[0, \tau]$, then a bound on the interval $[0,2 \tau]$, and finally a bound on $[0,+\infty)$. Let us then introduce such sequence of bounds as functions of the nonnegative real-valued parameter $R$, the radius of the ball of initial conditions:

$$
\begin{aligned}
& \alpha(R)=\kappa_{1}^{-1}\left(\mathrm{e}^{\kappa_{4}(R) \tau}\left(\kappa_{2}(R)+1\right)-1\right), \\
& \gamma(R)=\alpha(\alpha(R)), \\
& \omega(R)=\kappa_{1}^{-1}\left(\kappa_{2}(\gamma(R))+\frac{\tau}{4} \sup _{|a| \leq \gamma(R)} W(a)\right)+R,
\end{aligned}
$$

where $\kappa_{1}, \kappa_{2}, \kappa_{4}$ are the class $\mathcal{K}_{\infty}$ functions defined in Sect. 3.1. Observe that the functions $\alpha, \gamma, \omega$ are continuous and for all $R \geq 0$, the inequalities

$$
\omega(R) \geq \gamma(R) \geq \alpha(R) \geq R
$$

are satisfied. It will be proven below that $|x(t)| \leq \omega(R)$ for all $t \geq-2 \tau$. Define

$$
u_{0}(R)=\sup _{|a| \leq \omega(R)}|z(a)|+1
$$

and let $u_{0} \geq u_{0}(R)$. Having defined $u_{0}$ we can proceed with the rest of the proof. 
Consider the solution $x(t)$ of (14) with an initial condition $\varphi \in C^{1}\left([-2 \tau, 0], \mathbb{R}^{n}\right)$ such that $\|\varphi\|_{c} \leq R$. Let us show first that this solution is defined over $[-2 \tau, \tau]$. To prove this, let us proceed by contradiction. Suppose it is not defined over $[-2 \tau, \tau]$. Observe that, since $\|\varphi\|_{c} \leq R$, then $|z(\varphi(t-\tau))|<(1-\delta)^{-1} u_{0}$ for all $t \in[0, \tau]$, by definition of $u_{0}(\cdot)$ (see (49)). Hence, $\Psi(z(\varphi(t-\tau))$ ) is well-defined for all $t \in[0, \tau]$. Next, we deduce that, necessarily there exists $T \in(0, \tau]$ such that the solution exists for all $t \in[0, T)$. Such solution satisfies, for all $t \in[0, T)$ such that $t \in\left[t_{i}, t_{i+1}\right)$, $i=0,1, \ldots, k$, the differential inclusion

$$
\dot{x}(t) \in f(x(t))+g(x(t)) K(\Psi(z(\varphi(t-\tau)))),
$$

where $K(\Psi(z(\varphi(t-\tau))))$ denotes the set (12) with $u=z(\varphi(t-\tau))$. For all $t \in[0, T)$ such that $t \in\left[t_{i}, t_{i+1}\right), i=0,1, \ldots, k$, we are interested in finding an upper bound for the term

$$
L_{f} V(x(t))+L_{g} V(x(t)) v
$$

for any $v \in K(\Psi(z(\varphi(t-\tau))))$. Indeed, since for any $t \in\left[t_{i}, t_{i+1}\right), i=0,1, \ldots, k$, the derivative of $V$ along the trajectories of the system we consider satisfies

$$
\dot{V}(t)=L_{f} V(x(t))+L_{g} V(x(t)) \Psi(z(\varphi(t-\tau)))
$$

then

$$
\dot{V}(t)=L_{f} V(x(t))+L_{g} V(x(t)) v
$$

for some $v \in K(\Psi(z(\varphi(t-\tau))))$, and finding an upper bound for (51) means providing an upper bound for $\dot{V}(t)$. Observe that to find an upper bound for (51), it suffices to find an upper bound for

$$
\begin{aligned}
v_{a}:= & L_{f} V(x(t))+L_{g} V(x(t))(1+\lambda \delta) z(\varphi(t-\tau)) \\
& +L_{g} V(x(t))(\lambda-1) z(\varphi(t-\tau)) \varrho
\end{aligned}
$$

for all $t \in[0, T)$, with $\lambda$ any number in the interval $[-1,1]$ and where $\varrho=1$ or 0 . As a matter of fact, since in (51), $v \in K(\Psi(z(\varphi(t-\tau))))$, then, by (12), either

$$
v=(1+\lambda \delta) z(\varphi(t-\tau)), \text { with } \lambda \in[-1,1]
$$

(provided that $(1+\delta)^{-1} u_{j}<|z(\varphi(t-\tau))| \leq(1-\delta)^{-1} u_{0}$, in which case $\varrho=0$ ) or

$$
v=\lambda(1+\delta) z(\varphi(t-\tau)), \text { with } \lambda \in[0,1]
$$

(provided that $|z(\varphi(t-\tau))| \leq(1+\delta)^{-1} u_{j}$, in which case $\varrho=1$ ).

Hence, for a fixed $t$, the set of values in (51) obtained as $v$ ranges over $K(\Psi$ $(z(\varphi(t-\tau))))$ is contained in the set of values of $v_{a}$ as $\lambda \in[-1,1]$ and $\varrho \in\{0,1\}$. 
Now, adding and subtracting $L_{g} V(x(t))(1+\lambda \delta) z(x(t))$ on the right-hand side of the equality (52), and taking advantage of (15), we deduce that

$$
\begin{aligned}
v_{a} \leq & -W(x(t))+L_{g} V(x(t))(1+\lambda \delta)[z(\varphi(t-\tau))-z(x(t))] \\
& +L_{g} V(x(t))(\lambda-1) z(\varphi(t-\tau)) \varrho .
\end{aligned}
$$

Since $\delta \in(0,1)$, for any $t \in[0, T)$ and $\lambda \in[-1,1]$, the quantity $L_{g} V(x(t))$ $(1+\lambda \delta)[z(\varphi(t-\tau))-z(x(t))]$ belongs to the set

$$
\left\{L_{g} V(x(t))(1+\lambda)[z(\varphi(t-\tau))-z(x(t))], \lambda \in[-1,1]\right\} .
$$

Hence, if one finds a bound for

$$
\begin{aligned}
& -W(x(t))+L_{g} V(x(t))(1+\lambda)[z(\varphi(t-\tau))-z(x(t))] \\
& +L_{g} V(x(t))(\lambda-1) z(\varphi(t-\tau)) \varrho,
\end{aligned}
$$

then one also finds a bound for $v_{a}$.

Now, inequality (17) in Assumption (A3) implies that

$$
-\frac{1}{2} W(x(t))+L_{g} V(x(t))(1+\lambda \delta)[z(\varphi(t-\tau))-z(x(t))] \leq \kappa_{3}(R)[V(x(t))+1]
$$

and that, for all $\lambda_{a} \in[-1,1]$,

$$
-\frac{1}{2} W(x)+L_{g} V(x)\left(1+\lambda_{a}\right)[-z(x)] \leq \kappa_{3}(R)[V(x)+1] .
$$

Therefore, for all $\lambda \in[-1,1]$,

$$
-\frac{1}{2} W(x(t))+L_{g} V(x(t))(-1+\lambda) z(x(t)) \varrho \leq \kappa_{3}(R)[V(x(t))+1]
$$

with $\varrho=1$ or 0 . Next, from (54), (55) and (56), we deduce that

$$
\begin{aligned}
-W(x(t))+L_{g} V(x(t))(1+\lambda)[z(\varphi(t-\tau))-z(x(t))] \\
\quad+L_{g} V(x(t))(\lambda-1) z(\varphi(t-\tau)) \varrho \\
\leq 2 \kappa_{3}(R)[V(x(t))+1]=\kappa_{4}(R)[V(x(t))+1],
\end{aligned}
$$

and, therefore,

$$
v_{a} \leq \kappa_{4}(R)[V(x(t))+1]
$$

We deduce that necessarily, for all $t \in[0, T)$ such that $t \in\left[t_{i}, t_{i+1}\right), i=0,1, \ldots, k$, (i.e. at all inter-switching times) we have

$$
\dot{V}(t) \leq \kappa_{4}(R)[V(x(t))+1] .
$$


On the other hand, for any $t_{i} \in[0, T)$, with $i=0,1, \ldots, k$, (i.e. at the switching times)

$$
V\left(x\left(t_{i}^{+}\right)\right)=V\left(x\left(t_{i}\right)\right) .
$$

We conclude as in [14] that no finite escape time can exist. Indeed, for any $t \in[0, T)$, let $t \in\left[t_{i}, t_{i+1}\right)$ for some $i$. Then, integrating (58) from $t_{i}$ to $t$, we obtain

$$
V(x(t))+1 \leq \mathrm{e}^{\kappa_{4}(R)\left(t-t_{i}\right)}\left(V\left(x\left(t_{i}^{+}\right)\right)+1\right)=\mathrm{e}^{\kappa_{4}(R)\left(t-t_{i}\right)}\left(V\left(x\left(t_{i}\right)\right)+1\right),
$$

where the latter equality follows from (59). Similarly

$$
\begin{aligned}
V\left(x\left(t_{i}\right)\right)+1 & \leq \mathrm{e}^{\kappa_{4}(R)\left(t_{i}-t_{i-1}\right)}\left(V\left(x\left(t_{i-1}^{+}\right)\right)+1\right) \\
& =\mathrm{e}^{\kappa_{4}(R)\left(t_{i}-t_{i-1}\right)}\left(V\left(x\left(t_{i-1}\right)\right)+1\right) \\
& \leq \mathrm{e}^{\kappa_{4}(R)\left(t_{i}-t_{i-1}\right)} \mathrm{e}^{\kappa_{4}(R)\left(t_{i-1}-t_{i-2}\right)}\left(V\left(x\left(t_{i-2}^{+}\right)\right)+1\right) \\
& =\mathrm{e}^{\kappa_{4}(R)\left(t_{i}-t_{i-1}\right)} \mathrm{e}^{\kappa_{4}(R)\left(t_{i-1}-t_{i-2}\right)}\left(V\left(x\left(t_{i-2}\right)\right)+1\right) \\
& \vdots \\
& \leq \mathrm{e}^{\kappa_{4}(R)\left(t_{i}-t_{i-1}\right)} \mathrm{e}^{\kappa_{4}(R)\left(t_{i-1}-t_{i-2}\right)} \ldots \mathrm{e}^{\kappa_{4}(R)\left(t_{1}-t_{0}\right)}\left(V\left(x\left(t_{0}\right)\right)+1\right) \\
& \leq \mathrm{e}^{\kappa_{4}(R)\left(t_{i}-t_{0}\right)}\left(V\left(x\left(t_{0}\right)\right)+1\right) .
\end{aligned}
$$

Recalling that $t_{0}=0$, it follows that:

$$
V(x(t))+1 \leq \mathrm{e}^{\kappa_{4}(R) t}(V(x(0))+1),
$$

which shows that no finite escape time can actually exist. This fact and

$$
|z(\varphi(t-\tau))|<(1-\delta)^{-1} u_{0}(R), \forall t \in[0, \tau]
$$

imply that $x(t)$ can be extended beyond $T$. This yields a contradiction with the definition of $T$. It follows that $x(t)$ is defined for all $t \in[0, \tau]$. As before, by integrating (58) and bearing in mind (59), we infer that, for all $t \in[0, \tau]$,

$$
V(x(t))+1 \leq \mathrm{e}^{\kappa_{4}(R) t}[V(x(0))+1] .
$$

It follows immediately from (15) that, for all $t \in[0, \tau]$,

$$
\begin{aligned}
\kappa_{1}(|x(t)|) & \leq \mathrm{e}^{\kappa_{4}(R) \tau}\left[\kappa_{2}(|x(0)|)+1\right]-1 \\
& \leq \mathrm{e}^{\kappa_{4}(R) \tau}\left[\kappa_{2}(R)+1\right]-1 .
\end{aligned}
$$

It follows that, for all $t \in[0, \tau]$,

$$
|x(t)| \leq \alpha(R)
$$


where $\alpha(\cdot)$ is the function defined in (45). Observe that (63) and the inequality $\|\varphi\|_{c} \leq$ $R$ imply that, for all $t \in[\tau, 2 \tau],|z(x(t-\tau))| \leq \sup _{|a| \leq \alpha(R)}|z(a)|$. Since $\omega(\cdot) \geq \alpha(\cdot)$, it follows that, for all $t \in[\tau, 2 \tau]$,

$$
|z(x(t-\tau))|<(1-\delta)^{-1} u_{0}(R) .
$$

Hence, $\Psi(z(x(t-\tau)))$ is well-defined for all $t \in[\tau, 2 \tau]$. Moreover, the time derivative of $z(x(t-\tau))$, namely

$$
\frac{d}{d t} z(x(t-\tau))=\left.\frac{\partial z(x)}{\partial x}\right|_{x=x(t-\tau)}[f(x(t-\tau))+g(x(t-\tau)) \Psi(z(\varphi(t-2 \tau)))]
$$

is bounded for all $t \in[\tau, 2 \tau]$, hence the length of the inter-switching intervals is bounded away from zero on $[\tau, 2 \tau]$, and therefore, the switching times in that interval do not accumulate in finite time. Next, arguing exactly as before one can prove that $x(t)$ is defined for all $t \in[\tau, 2 \tau]$ and that, for all $t \in[\tau, 2 \tau]$,

$$
|x(t)| \leq \alpha(\alpha(R))=\gamma(R) .
$$

\subsection{Extending solutions for $t>2 \tau$}

To extend further the solution, we proceed by contradiction.

Let us assume that

$$
\sup \{t: x(s) \text { exists and }|x(s)| \leq \omega(R), \quad \forall s \in[-2 \tau, t]\}
$$

is a finite real number that we denote again $T$. From the inequality $\|\varphi\|_{c} \leq R,(63)$ and (65) and the facts that $R>0$ and $W$ is positive definite, we deduce that $T>2 \tau$. Next, observe that the continuity of the solutions and the definition of $u_{0}(\cdot)$ in (49) and (48) imply that, for all $t \in[-2 \tau, T)$,

$$
|z(x(t))| \leq \sup _{|a| \leq \omega(R)}\{|z(a)|\}<\frac{u_{0}(R)}{1-\delta} .
$$

We exploit this inequality to derive first an upper bound for $\dot{V}(t)$ and later on for $\dot{\mathcal{U}}(t)$, where $\mathcal{U}$ is a Lyapunov-Krasowskii functional to be introduced below.

Arguing as before (see (52) and the sentence following it), we claim that to find an upper bound for $\dot{V}(t)$, we need to find an upper bound to the expression below for all $t \in[2 \tau, T)$,

$$
\begin{aligned}
v_{b}(t):= & L_{f} V(x(t))+L_{g} V(x(t))(1+\lambda \delta) z(x(t-\tau)) \\
& +L_{g} V(x(t))(\lambda-1) z(x(t-\tau)) \varrho
\end{aligned}
$$

with $\lambda$ any number in the interval $[-1,1]$ and $\varrho \in\{0,1\}$. Recall that $\varrho=1$ if and only if $|z(x(t-\tau))| \leq(1+\delta)^{-1} u_{j}$. Thanks to Assumption (A1), we deduce that, for all 
$t \in[2 \tau, T)$,

$$
\begin{aligned}
v_{b}(t) \leq & -W(x(t))+L_{g} V(x(t))(1+\lambda \delta)[z(x(t-\tau))-z(x(t))] \\
& +L_{g} V(x(t))(\lambda-1) z(x(t-\tau)) \varrho .
\end{aligned}
$$

We now set $z(x(t-\tau))-z(x(t))$ in a form which allows us to use Assumption (A2). Let $t_{i j}, j=1, \ldots, k$ be the switching times in the interval $(t-\tau, t)$ and set without loss of generality $t_{i_{0}}=t-\tau, t_{i_{k+1}}=t$. We observe as before that the switching times do not accumulate in finite time. Hence we can write

$$
\begin{aligned}
& z(x(t))-z(x(t-\tau)) \\
& \quad=z(x(t))-z\left(x\left(t_{i_{k}}\right)\right)+z\left(x\left(t_{i_{k}}\right)\right) \ldots-z\left(x\left(t_{i_{1}}\right)\right)+z\left(x\left(t_{i_{1}}\right)\right)-z(x(t-\tau)) \\
& \quad=\sum_{j=0}^{k}\left[z\left(x\left(t_{i_{j+1}}\right)\right)-z\left(x\left(t_{i_{j}}\right)\right)\right] .
\end{aligned}
$$

For each $m \in\left[t_{i_{j}}, t_{i_{j+1}}\right), j=0,1, \ldots, k$,

$$
\begin{aligned}
z\left(x\left(t_{i_{j+1}}\right)\right)-z\left(x\left(t_{i_{j}}\right)\right) & \\
= & \int_{t_{i_{j}}}^{t_{i_{j+1}}} \frac{\partial z}{\partial x}(x(m))\left[f(x(m))+g(x(m)) \Psi\left(z\left(x\left(\left(t_{i_{j}}-\tau\right)^{+}\right)\right)\right)\right] d m \\
= & \int_{t_{i_{j}}}^{t_{i_{j+1}}} \frac{\partial z}{\partial x}(x(m))[f(x(m))+g(x(m)) \Psi(z(x(m-\tau)))] d m \\
= & \int_{t_{i_{j}}}^{t_{i_{j+1}}} \frac{\partial z}{\partial x}(x(m))\left[f(x(m))+g(x(m)) \psi_{1}(m-\tau) z(x(m-\tau))\right] d m
\end{aligned}
$$

where $^{3} \psi_{1}(\cdot)$ is a class $C^{1}$ function taking value in $[1-\delta, 1+\delta]$. Overall we have

3 Let $\Psi(z(x(m-\tau)))=\tilde{u}_{i}$ for $m \in\left[t_{i_{j}}, t_{i_{j+1}}\right)$, where $\tilde{u}_{i}=u_{i}$ or $\tilde{u}_{i}=u_{i}(1+\delta)^{-1}$. Then $(1+\delta)^{-1} \tilde{u}_{i}<$ $z(x(m-\tau)) \leq(1-\delta)^{-1} \tilde{u}_{i}$. At each $m \in\left[t_{i_{j}}, t_{i_{j+1}}\right), z(x(m-\tau))=\alpha(m-\tau)(1+\delta)^{-1} \tilde{u}_{i}+(1-\alpha(m-$ $\tau))(1-\delta)^{-1} \tilde{u}_{i}$, where $\alpha(\cdot)$ takes value in $[0,1]$. Observe also that, since $(1+\delta)^{-1} \tilde{u}_{i},(1-\delta)^{-1} \tilde{u}_{i}$ are constants, and $z(x(m-\tau))$ is a class $C^{1}$ function on the interval $\left[t_{i_{j}}, t_{i_{j+1}}\right)$, so is $\alpha(m-\tau)$. Moreover,

$$
\Psi(z(x(m-\tau)))=\tilde{u}_{i}=\left[\alpha(m-\tau)(1+\delta)^{-1}+(1-\alpha(m-\tau))(1-\delta)^{-1}\right]^{-1} z(x(m-\tau)),
$$

where the function $\psi_{1}(m-\tau)=\left[\alpha(m-\tau)(1+\delta)^{-1}+(1-\alpha(m-\tau))(1-\delta)^{-1}\right]^{-1}$ is a class $C^{1}$ function which spans the interval $[1-\delta, 1+\delta]$. Similar considerations hold when $\tilde{u}_{i}$ is negative or equal to zero. 


$$
\begin{aligned}
& z(x(t))-z(x(t-\tau)) \\
& \quad=\int_{t-\tau}^{t} \frac{\partial z}{\partial x}(x(m))\left[f(x(m))+g(x(m)) \psi_{2}(m-\tau) z(x(m-\tau))\right] d m
\end{aligned}
$$

with $\psi_{2}(\cdot):[t-2 \tau, t-\tau] \rightarrow[1-\delta, 1+\delta]$ a class $\bar{C}^{0}$ function, and hence

$$
\begin{aligned}
v_{b}(t) \leq & -W(x(t))+L_{g} V(x(t))(1+\lambda \delta) \int_{t-\tau}^{t} \frac{\partial z}{\partial x}(x(m))[f(x(m)) \\
& \left.+g(x(m)) \psi_{2}(m-\tau) z(x(m-\tau))\right] d m+\frac{\partial V}{\partial x} g(x(t))(\lambda-1) z(x(t-\tau)) \varrho .
\end{aligned}
$$

We deduce from Assumption (A2), that we have, for all $t \in[2 \tau, T)$,

$$
\begin{aligned}
v_{b}(t) \leq & -\frac{3}{4} W(x(t))+\frac{1}{\Omega} \int_{t-2 \tau}^{t} W(x(\ell)) \mathrm{d} \ell \\
& +L_{g} V(x(t))(\lambda-1) z(x(t-\tau)) \varrho .
\end{aligned}
$$

Since $\varrho=1$ if and only if $|z(x(t-\tau))| \leq(1+\delta)^{-1} u_{j}$, we have that, for all $t \in[2 \tau, T)$,

$$
v_{b}(t) \leq-\frac{3}{4} W(x(t))+\frac{1}{\Omega} \int_{t-2 \tau}^{t} W(x(\ell)) \mathrm{d} \ell+2\left|L_{g} V(x(t))\right|(1+\delta)^{-1} u_{j}
$$

and therefore

$$
\dot{V}(t) \leq-\frac{3}{4} W(x(t))+\frac{1}{\Omega} \int_{t-2 \tau}^{t} W(x(\ell)) \mathrm{d} \ell+2\left|L_{g} V(x(t))\right|(1+\delta)^{-1} u_{j}
$$

Next, with an abuse of notation, we define the following Lyapunov-Krasowskii functional

$$
\mathcal{U}(t)=V(x(t))+\frac{1}{8 \tau} \int_{t-2 \tau}^{t} \int_{s}^{t} W(x(\ell)) \mathrm{d} \ell \mathrm{d} s
$$


We deduce from (71) that, for all $t \in[2 \tau, T)$, the derivative of $\mathcal{U}$ along the trajectories of the system we consider satisfies

$$
\begin{aligned}
\dot{\mathcal{U}}(t) & \leq-\frac{1}{2} W(x(t))+\left(\frac{1}{\Omega}-\frac{1}{8 \tau}\right) \int_{t-2 \tau}^{t} W(x(\ell)) \mathrm{d} \ell+2\left|L_{g} V(x(t))\right|(1+\delta)^{-1} u_{j} \\
& \leq-\frac{1}{2} W(x(t))-\frac{1}{16 \tau} \int_{t-2 \tau}^{t} W(x(\ell)) \mathrm{d} \ell+2\left|L_{g} V(x(t))\right|(1+\delta)^{-1} u_{j},
\end{aligned}
$$

where the last inequality is a consequence of the condition $\Omega \geq 16 \tau$. Let $\kappa_{5}$ be a positive increasing function of class $C^{1}$ such that for all $x \in \mathbb{R}^{n}$

$$
\left|L_{g} V(x)\right| \leq \kappa_{5}(V(x))
$$

and set $\kappa_{6}(R)=\kappa_{5}\left(\kappa_{2}(\omega(R))\right)$ (observe that $\kappa_{6}$ is continuous). From (74), (15), the definition of $\kappa_{6}$ and the definition of $T$, we infer that, for all $t \in[2 \tau, T)$,

$$
\begin{aligned}
\dot{\mathcal{U}}(t) & \leq-\frac{1}{2} W(x(t))-\frac{1}{16 \tau} \int_{t-2 \tau}^{t} W(x(\ell)) \mathrm{d} \ell+2 \kappa_{6}(R)(1+\delta)^{-1} u_{j} \\
& \leq-\frac{1}{2} W(x(t))-\frac{1}{32 \tau^{2}} \int_{t-2 \tau}^{t} \int_{s}^{t} W(x(\ell)) \mathrm{d} \ell \mathrm{d} s+2 \kappa_{6}(R)(1+\delta)^{-1} u_{j} .
\end{aligned}
$$

Next, we would like to express the first two terms on the right-hand side of the last inequality in terms of $\mathcal{U}(t)$. This is possible according to Lemma 2 in Appendix A. Namely, one can determine a $C^{1}$ class- $\mathcal{K}_{\infty}$ function $\kappa_{7 \tau}$ and a function $\kappa_{8 \tau}$ of class $C^{1}$, positive and nondecreasing such that, for all $x \in \mathbb{R}^{n}$ and $z \geq 0$,

$$
\frac{\kappa_{7 \tau}(V(x)+z)}{\kappa_{8 \tau}(V(x)+z)} \leq \frac{1}{2} W(x)+\frac{1}{4 \tau} z
$$

where $z=\frac{1}{8 \tau} \int_{t-2 \tau}^{t} \int_{s}^{t} W(x(\ell)) \mathrm{d} \ell \mathrm{d} s$. From (76), it is possible to deduce that, for all $t \in[2 \tau, T)$,

$$
\dot{\mathcal{U}}(t) \leq-\frac{\kappa_{7 \tau}(\mathcal{U}(t))}{\kappa_{8 \tau}(\mathcal{U}(t))}+2 \kappa_{6}(R)(1+\delta)^{-1} u_{j}
$$

Since, for all $t \in[2 \tau, T),|x(t)| \leq \omega(R)$, we deduce that for all $t \in[2 \tau, T), V(x(t)) \leq$ $\kappa_{2}(\omega(R))$ and $\int_{t-2 \tau}^{t} \int_{s}^{t} W(x(\ell)) \mathrm{d} \ell \mathrm{d} s \leq 2 \tau^{2} \sup _{|a| \leq \omega(R)} W(a)$. It follows that

$$
\mathcal{U}(t) \leq \kappa_{2}(\omega(R))+2 \tau^{2} \sup _{|a| \leq \omega(R)} W(a)=: \kappa_{9}(R)
$$


where $\kappa_{9}$ is continuous and nondecreasing. Next, let us prove that, for all $t \in[2 \tau, T)$, the inequality

$$
\mathcal{U}(t) \leq \kappa_{2}(\gamma(R))+\frac{\tau}{4} \sup _{|a| \leq \gamma(R)} W(a)
$$

is satisfied. This result is the consequence of (77) and the fact that $\mathcal{U}(2 \tau) \leq \kappa_{2}(\gamma(R))+$ $\frac{\tau}{4} \sup _{|a| \leq \gamma(R)} W(a)$ and

$$
\dot{\mathcal{U}}(t)<0
$$

when $\mathcal{U}(t)=\kappa_{2}(\gamma(R))+\frac{\tau}{4} \sup _{|a| \leq \gamma(R)} W(a)$ provided that $u_{j}$ is appropriately chosen.

To see this, observe in particular (recall (3)) that $u_{j}$ can be made small by increasing the number of quantization levels $j$. Namely, let

$$
j \geq\left|\left(\log \frac{\mu(\varepsilon, R)(1+\delta)}{u_{0}(R)}\right)\left(\log \frac{1-\delta}{1+\delta}\right)^{-1}\right|+1
$$

where $\mu$ is continuous and such that, for all the real numbers $\varepsilon>0, R>0$,

$$
0<\mu(\varepsilon, R) \leq \min \left\{A_{1}(R), A_{2}(\varepsilon, R)\right\}
$$

with

$$
\begin{aligned}
A_{1}(R) & =\frac{\kappa_{7 \tau}\left(\kappa_{2}(\gamma(R))+\frac{\tau}{4} \sup _{|a| \leq \gamma(R)} W(a)\right)}{4 \kappa_{6}(R) \kappa_{8 \tau}\left(\kappa_{2}(\gamma(R))+\frac{\tau}{4} \sup _{|a| \leq \gamma(R)} W(a)\right)}, \\
A_{2}(\varepsilon, R) & =\frac{\kappa_{7 \tau}\left(\kappa_{1}(\varepsilon)\right)}{4 \kappa_{6}(R) \kappa_{8 \tau}\left(\kappa_{2}(\gamma(R))+\frac{\tau}{4} \sup _{|a| \leq \gamma(R)} W(a)\right)} .
\end{aligned}
$$

Observe that (81) and (3) imply

$$
(1+\delta)^{-1} u_{j} \leq \mu(\varepsilon, R) .
$$

Hence, (77) rewrites as

$$
\dot{\mathcal{U}}(t) \leq-\frac{\kappa_{7 \tau}(\mathcal{U}(t))}{\kappa_{8 \tau}(\mathcal{U}(t))}+2 \kappa_{6}(R) \mu(\varepsilon, R)
$$

From this, since $\mu(\varepsilon, R) \leq A_{1}(\varepsilon, R)$, it is immediate to see that when $\mathcal{U}(t)=\kappa_{2}(\gamma(R))+\frac{\tau}{4} \sup _{|a| \leq \gamma(R)} W(a),(80)$ is satisfied and (79) holds.

From (79), it follows immediately that, for all $t \in[2 \tau, T)$,

$$
|x(t)| \leq \kappa_{1}^{-1}\left(\kappa_{2}(\gamma(R))+\frac{\tau}{4} \sup _{|a| \leq \gamma(R)} W(a)\right) .
$$


We deduce from (47) and $R>0$ that, for all $t \in[2 \tau, T)$,

$$
|x(t)|<\omega(R) \text {. }
$$

This inequality and (67) imply that $x(t)$ can be extended beyond $T$. This yields a contradiction with the definition of $T$. We deduce that $x(t)$ is defined over $[-2 \tau,+\infty)$ and bounded in norm by $\omega(R)$.

\subsection{Practical convergence}

Observe that, arguing as before, one can prove that for all $t \geq 2 \tau$, we have

$$
\begin{aligned}
\dot{\mathcal{U}}(t) & \leq-\frac{\kappa_{7 \tau}(\mathcal{U}(t))}{\kappa_{8 \tau}(\mathcal{U}(t))}+2 \mu(\varepsilon, R) \kappa_{6}(R) \\
& \leq-\frac{\kappa_{7 \tau}(\mathcal{U}(t))}{\kappa_{8 \tau}\left(\kappa_{2}(\gamma(R))+\frac{\tau}{4} \sup _{|a| \leq \gamma(R)} W(a)\right)}+2 \mu(\varepsilon, R) \kappa_{6}(R) .
\end{aligned}
$$

Since, according to (82),

$$
4 \mu(\varepsilon, R) \kappa_{6}(R) \leq \frac{\kappa_{7 \tau}\left(\kappa_{1}(\varepsilon)\right)}{\kappa_{8 \tau}\left(\kappa_{2}(\gamma(R))+\frac{\tau}{4} \sup _{|a| \leq \gamma(R)} W(a)\right)}
$$

we deduce that, there exists $t_{L} \geq 0$ such that, for all $t \geq t_{L}$, the inequality

$$
\mathcal{U}(t) \leq \kappa_{1}(\varepsilon)
$$

is satisfied. It follows that, for all $t \geq t_{L}$,

$$
|x(t)| \leq \varepsilon
$$

that is the thesis.

Remark 5 It has been observed in the proof that, by the definition (66) of $T$, for all $t \in[2 \tau, T),|x(t)|<\omega(R)$, and therefore $\frac{1}{8 \tau} \int_{t-2 \tau}^{t} \int_{s}^{t} W(x(\ell)) \mathrm{d} \ell \mathrm{d} s \leq \frac{\tau}{4} \sup _{|a| \leq \omega(R)}$ $W(a)$. Set:

$$
\kappa_{7 \tau}^{R}(\xi)=\frac{\kappa_{7 \tau}(\xi)}{\kappa_{8 \tau}\left(\kappa_{2}(\omega(R))+\frac{\tau}{4} \sup _{|a| \leq \omega(R)} W(a)\right)} .
$$

Then, one can use in the proof the inequality

$$
\dot{\mathcal{U}}(t) \leq-\kappa_{7 \tau}^{R}(\mathcal{U}(t))+2 \mu(\varepsilon, R) \kappa_{6}(R)
$$

instead of (77). In particular one can follow exactly the same passages as before, provided that in the definition (82) of $\mu(\varepsilon, R)$, the functions $A_{1}(R)$ and $A_{2}(\varepsilon, R)$ in (83) 
are defined as

$$
\begin{aligned}
A_{1}(R) & =\frac{\kappa_{7 \tau}^{R}\left(\kappa_{2}(\gamma(R))+\frac{\tau}{4} \sup _{|a| \leq \gamma(R)} W(a)\right)}{4 \kappa_{6}(R)}, \\
A_{2}(\varepsilon, R) & =\frac{\kappa_{7 \tau}^{R}\left(\kappa_{1}(\varepsilon)\right)}{4 \kappa_{6}(R)} .
\end{aligned}
$$

Then, by replacing the differential inequality (88) with (93), and the inequality (89) with

$$
4 \mu(\varepsilon, R) \kappa_{6}(R) \leq \kappa_{7 \tau}^{R}\left(\kappa_{1}(\varepsilon)\right),
$$

we can again conclude that $x(t)$ enters the closed ball of radius $\varepsilon$ in finite time and remains in it thereafter.

This remark is useful to simplify the proof in the particular case where a constant function can be chosen for the function $\kappa_{8 \tau}$ in (76), for instance when the positive definite function $W(x)$ is lower bounded by a class- $\mathcal{K}_{\infty}$ function, as it happens when system (13) is linear.

Observe, finally, that a function $\kappa_{7 \tau}^{R}$ of class $\mathcal{K}$ such that (93) is satisfied can be found without necessarily relying on the knowledge of $\kappa_{7 \tau}$ and $\kappa_{8 \tau}$. In fact, bearing in mind (75), it suffices to find $\kappa_{7 \tau}^{R}$ such that

$$
\frac{1}{2} W(x(t))+\frac{1}{4 \tau} z(t) \geq \kappa_{7 \tau}^{R}(\mathcal{U}(t))
$$

with

$$
z(t)=\frac{1}{8 \tau} \int_{t-2 \tau}^{t} \int_{s}^{t} W(x(\ell)) \mathrm{d} \ell \mathrm{d} s .
$$

For instance, one can choose $\kappa_{7 \tau}^{R}(s)=K_{e}\left(\frac{1}{2} B_{S}(s)\right)$, where

$$
K_{e}(m)=\frac{1}{\bar{m}} \int_{0}^{m} \min _{l \leq|\xi| \leq \bar{m}} \mathcal{W}(\xi) d l, \quad m \in[0, \bar{m}]
$$

$\mathcal{W}(\xi)=\frac{1}{2} W(x)+\frac{1}{4 \tau} z, \xi=\left(x^{\mathrm{T}} z\right)^{\mathrm{T}}, \bar{m}=\omega(R)+z_{R}$ and $B_{S}$ is defined as

$$
B_{S}(l)=\min \left\{\kappa_{2}^{-1}\left(\frac{l}{2}\right), \frac{l}{2}\right\}
$$


As a matter of fact, for all $|\xi| \leq \bar{m}$,

$$
\mathcal{W}(\xi) \geq \min _{|\xi| \leq \bar{m}} \mathcal{W}(\xi) \geq \frac{1}{\bar{m}} \int_{0}^{|\xi|} \min _{|\xi| \leq \bar{m}} \mathcal{W}(\xi) d l \geq \frac{1}{\bar{m}} \int_{0}^{|\xi|} \min _{l \leq|\xi| \leq \bar{m}} \mathcal{W}(\xi) d l=K_{e}(|\xi|)
$$

Now, $|x|+z \leq 2|\xi|$ and

$$
|x|+z \geq \kappa_{2}^{-1}(V(x))+z \geq B_{S}(V(x)+z)
$$

with ${ }^{4} B_{S}(l)$ as in (96). Hence,

$$
\mathcal{W}(\xi) \geq K_{e}(|\xi|) \geq K_{e}\left(\frac{1}{2}(|x|+z)\right) \geq K_{e}\left(\frac{1}{2} B_{S}(V(x)+z)\right)=\kappa_{7 \tau}^{R}(V(x)+z)
$$

and, therefore,

$$
\frac{1}{2} W(x(t))+\frac{1}{4 \tau} z(t)=\mathcal{W}(\xi(t)) \geq \kappa_{7 \tau}^{R}(V(x(t))+z(t))=\kappa_{7 \tau}^{R}(U(t))
$$

as desired.

We could have stated the result directly in terms of the class- $\mathcal{K}$ function $\kappa_{7 \tau}^{R}$ just derived rather than introducing the two class- $\mathcal{K}_{\infty}$ functions $\kappa_{7 \tau}, \kappa_{8 \tau}$. We decided to adopt the latter in order not to have in $A_{1}(R), A_{2}(\varepsilon, R)$ (and hence in the conditions on the number of quantization levels $j$ ) a class- $\mathcal{K}$ function depending implicitly on the parameter $R$.

Remark 6 The proof is constructive: it gives the explicit expressions for the two design parameters $u_{0}$ (see (49)), and $j$ (see (81) and (82)).

\section{Conclusion}

We have presented a Lyapunov-Krasowskii functional approach to solve the problem of determining quantized feedbacks with delay which semi-globally practically stabilize the origin of nonlinear systems. For a fairly general family of systems, and given any value of the quantization density, we have characterized the maximal allowable constant delay which the closed-loop system can tolerate. A problem which in our opinion would be interesting to investigate is how, for systems with a well-defined relative degree, our result can be propagated via the backstepping technique.

4 Let $\alpha_{1}(r)=\kappa_{2}^{-1}(r), \alpha_{2}(r)=r, \alpha_{3}(r)=\min \left\{\alpha_{1}(r), \alpha_{2}(r)\right\}$, and $2 a=V(x), 2 b=z$. Then, bearing in mind that, for any function $\alpha$ of class $\mathcal{K}_{\infty}, \alpha(a+b) \leq \alpha(2 a)+\alpha(2 b)$, we have

$$
\alpha_{1}(2 a)+\alpha_{2}(2 b) \geq \alpha_{3}(2 a)+\alpha_{3}(2 b) \geq \alpha_{3}(a+b) .
$$

This proves $\kappa_{2}^{-1}(V(x))+z \geq B_{S}(V(x)+z)$. 
Acknowledgments The work of the first author is partially supported by PRIN 2009 Advanced methods for feedback control of uncertain nonlinear systems.

Open Access This article is distributed under the terms of the Creative Commons Attribution Noncommercial License which permits any noncommercial use, distribution, and reproduction in any medium, provided the original author(s) and source are credited.

\section{Appendix A: Technical lemmas}

Lemma 1 Let $\mathcal{W}: \mathbb{R}^{n} \rightarrow \mathbb{R}$ be a continuous and positive definite function. For all $m \geq 0$, let

$$
\begin{aligned}
& K_{a}(m)=\int_{0}^{\min \{m, 1\}} \min _{l \leq|\xi| \leq 1} \mathcal{W}(\xi) d l+\max \{0, m-1\}, \\
& K_{b}(m)=1+\frac{K_{a}(m)}{\min _{1 \leq|\xi| \leq \max \{1, m\}} \mathcal{W}(\xi)} .
\end{aligned}
$$

Then $K_{a}$ belongs to $\mathcal{K}_{\infty}, K_{b}$ is continuous, positive and increasing over $[0,+\infty)$ and, for all $X \in \mathbb{R}^{n}$,

$$
\frac{K_{a}(|X|)}{K_{b}(|X|)} \leq \mathcal{W}(X) .
$$

Proof The fact that $\mathcal{W}(X)$ is positive definite implies that both $K_{a}$ and $K_{b}$ are welldefined and continuous. Let us prove that $K_{a}$ belongs to $\mathcal{K}_{\infty}$. Observe that $K_{a}(0)=0$. When $m \in[0,1], K_{a}(m)=\int_{0}^{m} \min _{l \leq|\xi| \leq 1} \mathcal{W}(\xi) d l$. Therefore this function is increasing over $[0,1]$. When $m>1, K_{a}(m)=\int_{0}^{1} \min _{l \leq|\xi| \leq 1} \mathcal{W}(\xi) d l+m-1$. Therefore, this function is increasing over $[1,+\infty)$ and goes to the infinity when its argument does. Consequently, $K_{a}$ is of class $\mathcal{K}_{\infty}$. If follows that $K_{b}$ is a positive and increasing over $[0,+\infty)$.

Next, to establish (99), we distinguish between two cases.

First case: $|X| \leq 1$. Then $K_{a}(|X|)=\int_{0}^{|X|} \min _{l \leq|\xi| \leq 1} \mathcal{W}(\xi) d l \leq|X| \min _{|X| \leq|\xi| \leq 1}$ $\mathcal{W}(\xi) \leq \mathcal{W}(X)$. Moreover, $K_{b}(|X|) \geq 1$. It follows that $\frac{K_{a}(|X|)}{K_{b}(|X|)} \leq \mathcal{W}(X)$.

Second case: $|X| \geq 1$. Then

$$
\begin{aligned}
& K_{a}(|X|)=\int_{0}^{1} \min _{l \leq|\xi| \leq 1} \mathcal{W}(\xi) d l+|X|-1>0, \\
& K_{b}(|X|)=1+\frac{K_{a}(|X|)}{\min _{1 \leq|\xi| \leq|X|} \mathcal{W}(\xi)}>\frac{K_{a}(|X|)}{\min _{1 \leq|\xi| \leq|X|} \mathcal{W}(\xi)}>0 .
\end{aligned}
$$

Therefore,

$$
\min _{1 \leq|\xi| \leq|X|} \mathcal{W}(\xi)>\frac{K_{a}(|X|)}{K_{b}(|X|)}>0 .
$$


It follows that

$$
\mathcal{W}(X)>\frac{K_{a}(|X|)}{K_{b}(|X|)}>0 .
$$

Lemma 2 Let $\tau>0, W$ be a positive definite function. Then one can determine a function $K_{c}$ of class $\mathcal{K}_{\infty}$ and a function $K_{d}$, positive, continuous and increasing over $[0,+\infty)$ such that, for all $x \in \mathbb{R}^{n}$ and $z \geq 0$,

$$
\frac{K_{c}(V(x)+z)}{K_{d}(V(x)+z)} \leq \frac{1}{2} W(x)+\frac{1}{4 \tau} z .
$$

Proof First, observe that the inequalities (15) imply that for all $x \in \mathbb{R}^{n}, z \geq 0$,

$$
|x|+z \leq \kappa_{1}^{-1}(V(x))+z \leq B_{L}(V(x)+z)
$$

with

$$
B_{L}(l)=\kappa_{1}^{-1}(l)+l
$$

and

$$
|x|+z \geq \kappa_{2}^{-1}(V(x))+z \geq B_{S}(V(x)+z)
$$

with

$$
B_{S}(l)=\min \left\{\kappa_{2}^{-1}\left(\frac{l}{2}\right), \frac{l}{2}\right\} .
$$

From Lemma 1, it follows immediately that one can determine a function $K_{a}$ of class $\mathcal{K}_{\infty}$ and a function $K_{b}$, positive, continuous and increasing over $[0,+\infty)$ such that, for all $x \in \mathbb{R}^{n}$ and $z \geq 0$,

$$
\frac{K_{a}(|x|+z)}{K_{b}(|x|+z)} \leq \frac{1}{2} W(x)+\frac{1}{4 \tau} z .
$$

From this inequality, (104) and (97), it follows that

$$
\frac{K_{a}\left(B_{S}(V(x)+z)\right)}{K_{b}\left(B_{L}(V(x)+z)\right)} \leq \frac{1}{2} W(x)+\frac{1}{4 \tau} z .
$$

This allows us to conclude. 


\section{References}

1. Ceragioli F, De Persis C (2007) Discontinuous stabilization of nonlinear systems: Quantized and switching control. Syst Control Lett 56:461-473

2. De Persis C (2009) Robust stabilization of nonlinear systems by quantized and ternary control. Syst Control Lett 58(8):602-608

3. Elia N, Mitter SK (2001) Stabilization of linear systems with limited information. IEEE Trans Autom Control 46(9):1384-1400

4. Fridman E, Dambrine M, Yeganefar N (2008) On input-to-state stability of systems with time-delay: a matrix inequalities approach. Automatica 44:2364-2369

5. Hayakawa T, Ishii H, Tsumura K (2006) Adaptive quantized control for nonlinear uncertain systems. In: Proceedings of 2006 American control conference, Minneapolis, MN, USA

6. Hahn W (1967) Stability of motion. Springer, Berlin

7. Isidori A (1999) Nonlinear control systems, vol 2. Springer, London

8. Jankovic M (2001) Control-Lyapunov-Razumikhin functions and robust stabilization of time delay systems. IEEE Trans Autom Control 46(7):1048-1060

9. Karafyllis I (2006) Lyapunov theorems for systems described by retarded functional differential equations. Nonlinear Anal Theory Methods Appl 64(3):590-617

10. Khalil HK (1996) Nonlinear systems, 2nd edn. Prentice Hall, Upper Saddle River

11. Krasowskii NN (1963) Stability of motion. Stanford University Press, Stanford

12. Liberzon D (2003) Hybrid feedback stabilization of systems with quantized signals. Automatica 39(9):1543-1554

13. Liberzon D (2006) Quantization, time delays, and nonlinear stabilization. IEEE Trans Autom Control 51(7):1190-1195

14. Mazenc F, Bliman P-A (2006) Backstepping design for time-delay nonlinear systems. IEEE Trans Autom Control 51:149-154

15. Mazenc F, Mondié S, Francisco R (2004) Global asymptotic stabilization of feedforward systems with delay in the input. IEEE Trans Autom Control 49(5):844-850

16. Michiels W, Sepulchre R, Roose R (2001) Stability of perturbed delay differential equations and stabilization of nonlinear cascade systems. SIAM J Control Optim 40(3):661-680

17. Mazenc F, Niculescu S (2001) Lyapunov stability analysis for nonlinear delay systems. Syst Control Lett $42(4): 245-251$

18. Pepe P, Jiang ZP (2006) A Lyapunov-Krasovskii methodology for ISS and iISS of time-delay systems. Syst Control Lett 55(12):1006-1014

19. Sontag ED (1989) A "universal" construction of Artstein's theorem on nonlinear stabilization. Syst Control Lett 13:117-123

20. Teel AR (1998) Connections between Razumikhin-type theorems and the ISS nonlinear small-gain theorem. IEEE Trans Autom Control 43(7):960-964

21. Teel AR, Praly L (1996) Tool for semi-global stabilization by partial state and output feedback. SIAM J Control Optim 33:1443-1488 